\title{
A structural signature motif enlightens the origin and diversification of nuclear receptors
}

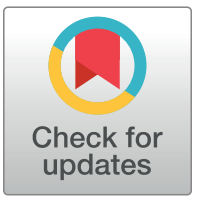

\section{G OPEnACCESS}

Citation: Beinsteiner B, Markov GV, Erb S, Chebaro Y, McEwen AG, Cianférani S, et al. (2021) A structural signature motif enlightens the origin and diversification of nuclear receptors. PLoS Genet 17(4): e1009492. https://doi.org/10.1371/journal. pgen.1009492

Editor: Artyom Kopp, University of California Davis, UNITED STATES

Received: December 14, 2020

Accepted: March 15, 2021

Published: April 21, 2021

Peer Review History: PLOS recognizes the benefits of transparency in the peer review process; therefore, we enable the publication of all of the content of peer review and author responses alongside final, published articles. The editorial history of this article is available here: https://doi.org/10.1371/journal.pgen.1009492

Copyright: $\odot 2021$ Beinsteiner et al. This is an open access article distributed under the terms of the Creative Commons Attribution License, which permits unrestricted use, distribution, and reproduction in any medium, provided the original author and source are credited.

Data Availability Statement: The data and R script necessary to reproduce Fig 3 are available from Dryad (https://doi.org/10.5061/dryad.kkwh70s48). All other relevant data or accession numbers are

\author{
Brice Beinsteiner $\oplus^{1,2,3,4 \ddagger}$, Gabriel V. Markov $\oplus^{5 \ddagger}$, Stéphane Erb $\odot^{6}$, \\ Yassmine Chebaro $\mathbb{1}^{1,2,3,4}$, Alastair G. McEwen $\mathbb{1}^{1,2,3,4}$, Sarah Cianférani ${ }^{6}$, \\ Vincent Laudet $\bigoplus^{7 *}$, Dino Moras $\bigoplus^{1,2,3,4 *}$, Isabelle M. L. Billas $\oplus^{1,2,3,4 *}$
}

1 IGBMC (Institute of Genetics and of Molecular and Cellular Biology), Illkirch, France, 2 Université de Strasbourg, Unistra, Strasbourg, France, 3 Institut National de la Santé et de la Recherche Médicale (INSERM) U1258, Illkirch, France, 4 Centre National de la Recherche Scientifique (CNRS) UMR 7104, Illkirch, France, 5 Sorbonne Université, CNRS, UMR 8227, Integrative Biology of Marine Models, (LBI2M, UMR8227), Station Biologique de Roscoff (SBR), Roscoff, France, 6 Laboratoire de Spectrométrie de Masse BioOrganique, Université de Strasbourg, CNRS, IPHC UMR 7178, Strasbourg, France, 7 Marine Eco-EvoDevo Unit. Okinawa Institute of Science and Technology, Onna-son, Okinawa, Japan

$\ddagger$ co-first authors

*vincent.laudet@oist.jp (VL); moras@igbmc.fr (DM); billas@igbmc.fr (IMLB)

\section{Abstract}

Nuclear receptors are ligand-activated transcription factors that modulate gene regulatory networks from embryonic development to adult physiology and thus represent major targets for clinical interventions in many diseases. Most nuclear receptors function either as homodimers or as heterodimers. The dimerization is crucial for gene regulation by nuclear receptors, by extending the repertoire of binding sites in the promoters or the enhancers of target genes via combinatorial interactions. Here, we focused our attention on an unusual structural variation of the $\alpha$-helix, called $\pi$-turn that is present in helix $\mathrm{H} 7$ of the ligand-binding domain of RXR and HNF4. By tracing back the complex evolutionary history of the $\pi$-turn, we demonstrate that it was present ancestrally and then independently lost in several nuclear receptor lineages. Importantly, the evolutionary history of the $\pi$-turn motif is parallel to the evolutionary diversification of the nuclear receptor dimerization ability from ancestral homodimers to derived heterodimers. We then carried out structural and biophysical analyses, in particular through point mutation studies of key RXR signature residues and showed that this motif plays a critical role in the network of interactions stabilizing homodimers. We further showed that the m-turn was instrumental in allowing a flexible heterodimeric interface of RXR in order to accommodate multiple interfaces with numerous partners and critical for the emergence of high affinity receptors. Altogether, our work allows to identify a functional role for the m-turn in oligomerization of nuclear receptors and reveals how this motif is linked to the emergence of a critical biological function. We conclude that the $\pi$-turn can be viewed as a structural exaptation that has contributed to enlarging the functional repertoire of nuclear receptors. 
within the manuscript and its Supporting Information files.

Funding: This work was supported by the Agence Nationale de la Recherche [Grant Number ANR2010-BLAN-1234 01] (V.L., I.M.L.B, D.M.), by the Université de Strasbourg (Unistra) (I.M.L.B., D.M., S.E., A.G.M, B.B, Y.C.), CNRS (I.M.L.B., D.M., S.C., A.G.M, B.B, Y.C.) and INSERM (I.M.L.B., D.M., A.G. M, B.B, Y.C.), by the association Alsace contre le Cancer (I.M.L.B.). We thank the Fondation pour la Recherche Médicale [Grant number FRM FDT20170437233] and Eurostars for fellowships awarded to BB. Support and usage of platforms was provided by the French Infrastructure for Integrated Structural Biology [FRISBI, ANR-10INSB-05-01], Instruct-ERIC, a Landmark ESFRI project (I.M.L.B., D.M., A.G.M, B.B, Y.C.) and by the French Proteomic Infrastructure [ProFl, ANR10-INBS-08-03] (S.E, S.C.). Financial support was provided by GIS IBiSA and Région Alsace in purchasing a Synapt G2 HDMS instrument (S.E and S.C.). The funders played no role in the study design, data collection and analysis, decision to publish, or preparation of the manuscript.

Competing interests: The authors declare that they have no conflict of interest.

\section{Author summary}

The origin of novelties is a central topic in evolutionary biology. A fundamental question is how organisms constrained by natural selection can divert from existing schemes to set up novel structures or pathways. Among the most important strategies are exaptations, which represent pre-adaptation strategies. Many examples exist in biology, at both morphological and molecular levels, such as the one reported here that focuses on an unusual structural feature called the $\pi$-turn. It is found in the structure of the most ancestral nuclear receptors RXR and HNF4. The analyses trace back the complex evolutionary history of the $\pi$-turn to more than 500 million years ago, before the Cambrian explosion and show that this feature was essential for the heterodimerization capacity of RXR. Nuclear receptor lineages that emerged later in evolution lost the $\pi$-turn. We demonstrate here that this loss in nuclear receptors that heterodimerize with RXR was critical for the emergence of high affinity receptors, such as the vitamin $\mathrm{D}$ and the thyroid hormone receptors. On the other hand, the conserved $\pi$-turn in RXR allowed it to accommodate multiple heterodimer interfaces with numerous partners. This structural exaptation allowed for the remarkable diversification of nuclear receptors.

\section{Introduction}

The nuclear hormone receptor (NR) superfamily includes receptors for hydrophobic ligands such as steroid hormones, retinoic acids, thyroid hormones or fatty acids derivatives [1,2]. This superfamily, which clusters 48 genes in human, is subjected to an intense scrutiny because of the essential role played by NRs in animal development, metabolism and physiology. NRs are important drug targets since dysfunctions of homeostasis and signaling pathways controlled by these receptors are associated with many diseases including cancer, metabolic syndrome or reproductive failure [3].

All nuclear receptor proteins share a characteristic modular structure that consists of conserved DNA and ligand binding domains (DBD and LBD, respectively) separated and flanked by poorly conserved flexible regions [1,2]. Typically, distant NRs exhibit ca. $60 \%$ sequence identity in their DBD and $30 \%$ in their LBD. The availability of the ligand controls NR activity in space and in time since ligand binding inside a specific pocket within the LBD induces a conformational change of the receptor allowing the release of corepressors, the recruitment of coactivators and the transactivation of target genes [1,2].

Given their importance and also because their long conserved domains are favorable for phylogenetic analysis, the origin of the NR superfamily have been scrutinized for a long time, allowing to define distinct subfamilies [4-6]. Full NRs are specific to animals whereas DBD sequences have been found in the genomes of some choanoflagellates, the closest metazoan relatives [7]. After several lineage-specific events of gene loss or gene duplications, the size of the superfamily ranges from about 20 members in insects to about 48 to 70 in vertebrates, with a specific expansion in some lineages such as nematodes for which more than $260 \mathrm{NR}$ genes are present [8-10].

The analysis of complete genome sequences available in a number of animal species, including early metazoans such as sponges, placozoans or cnidarians have allowed a better understanding of the first step of NR diversification. The observation that sponges, which despite some controversy are believed to be the earliest metazoan phyla [11] contains only two NR genes, called here SpNR1 and SpNR2, have shed a decisive light on the first steps of NR evolution [9]. This has allowed the positioning of the root of the NR tree within subfamily II that in 
particular contains the retinoid X receptor (RXR), the hepatocyte nuclear factor 4 (HNF4) and the COUP Transcription Factor 1 (COUP-TF)) and therefore that cannot be considered as monophyletic. This view separates the family into HNF4 on the one hand and all the other NRs on the other hand (Fig 1A). This phylogeny of early NRs now enables the study of the diversification and evolution of the various functions of NRs, such as ligand binding, DNA binding or dimerization. This was done for ligand binding and it allowed to propose that the ancestral NR was a sensor molecule capable of binding fatty acids with low affinity and low selectivity $[9,12,13]$. However, the same kind of evolutionary analysis has not yet been carried out to study the dimerization properties, a critical aspect of the nuclear receptors functions.

Thanks to their dimerization capability, NRs expanded the range of DNA target sequences through which they regulate target gene expression $[1,2,14]$. Several distinct dimerization properties have been characterized in NRs among which homodimerization on either palindromic or direct repeat DNA sequences, heterodimerization with RXR as a common partner, and even monomer binding (that is absence of dimerization) through the binding to extended half-site response elements (as depicted in Fig 1B) [14]. The pivotal role of RXR (and of the insect homolog ultraspiracle protein (USP)) in this context has to be pointed out, as it is the promiscuous partner for more than 15 distinct high-affinity liganded NRs, including the retinoic acid receptor (RAR), the thyroid hormone receptor (TR), the vitamin D receptor (VDR), the peroxisome-proliferator-activated receptor (PPAR), the liver X receptor (LXR) or the ecdysone receptor $(\mathrm{EcR})$ in insects. Structural analysis revealed that NRs contain two separable dimerization interfaces, a relatively weak, albeit important interface in the DBD that plays a key role in DNA target site selection $[15,16]$ and another stronger interface in the LBD. The detailed analysis of the LBDs dimerization interface highlighted the rules controlling homoversus heterodimerization and allowed two functional NR classes to be defined according to their oligomeric behavior [17]. Class I NRs behave either as monomers or homodimers and exhibit a set of conserved residues that form a communication pathway linking helix 1 to the dimerization interface via helix 8 . In contrast class II receptors encompass all NRs that heterodimerize with RXR and exhibit a different communication pathway linking the central helices $\mathrm{H} 4 / \mathrm{H} 5$ to the dimerization interface via a conserved arginine residue in the loop between helices $\mathrm{H} 8$ and $\mathrm{H} 9$ [17]. To deepen our understanding of how changes in NR dimerization properties contributed to the diversification of the NR superfamily, we carried out an evolutionary analysis of NR genes, focusing on the evolution of dimerization across the entire NR superfamily. We show here that homodimeric binding was ancestral, whereas heterodimeric and monomeric behaviors evolved later. We further identified a specific structural feature present in helix H7, a so-called $\pi$-turn or $\alpha / \pi$-bulge, present in RXR and HNF4, as being an ancestral motif critical for the homodimerization of the most ancient NRs. We traced back the complex evolutionary history of this $\pi$-turn showing that it was instrumental in the origin of heterodimerization, by allowing a flexible dimerization surface of RXR to accommodate numerous partners with multiple interfaces. The $\pi$-turn was originally used for homodimerization, but later was utilized for a different function, namely heterodimerization. This can be considered as a structural exaptation which can be seen as instrumental for the expansion of the repertoire of NR functions.

\section{Results}

\section{A specific $\pi$-turn motif is an ancestral feature of helix $\mathrm{H} 7$}

Since the first crystal structure of a NR LBD $[18,19]$ more than 800 sets of LBD coordinates have been deposited in the Protein Data Bank. A comparative analysis of these structures with the entire PDB data base showed that the canonical $\alpha$-helical fold of the LBD is conserved, 
A

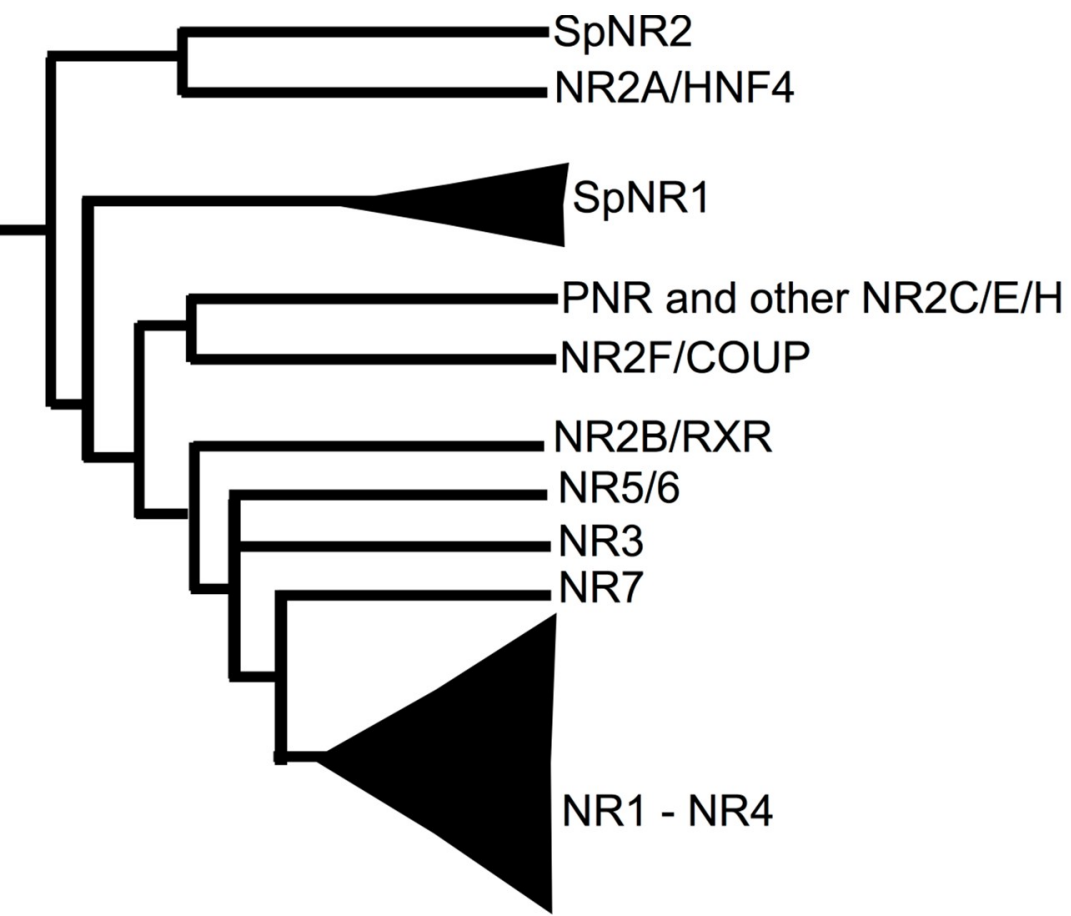

B

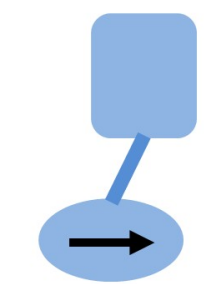

Half site

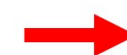

Monomer

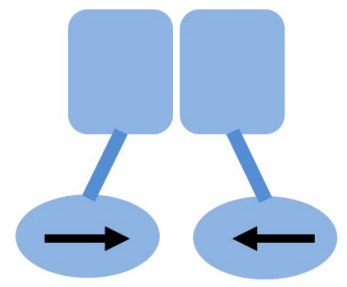

Palindrome

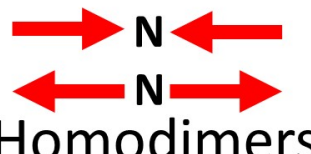

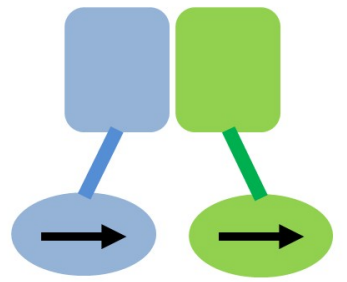

Direct repeat

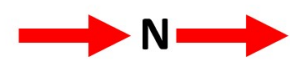

Heterodimers

Fig 1. (A) Simplified consensus tree of NR phylogeny based on Bridgham et al., 2010 [9]. (B) Some of the diversity found for the different DNA response elements (REs), illustrated here with a monomeric NR on an (extended) halfsite; a homodimer on an inverted or an everted RE; and finally a heterodimer on direct repeat RE; $\mathrm{N}$ is the number of base pairs in the spacer between the two half-sites.

https://doi.org/10.1371/journal.pgen.1009492.g001

suggesting strong structure-function constraints during evolution. A peculiar feature emerged from this structural analysis, which the presence of a helical deformation called $\pi$-turn or a $\alpha /$ $\pi$-bulge within the $\alpha$-helix 7 of RXR-USP and HNF4 LBDs (RXR, PDB: 1LBD, 6HN6 [18,19]; USP, PDB: 1G2N, [20]; HNF4), PDB: 1LV2, [21] (Fig 2). $\pi$-helices and $\pi$-turns account for over $15 \%$ of all known protein structures deposited in the PDB database [22-25]. The $\pi$-type helical structures are thermodynamically less stable than $\alpha$-helices and are considered to be favored only when they are associated with a functional advantage, typically for interactions with ligands or in the functioning of helical transmembrane domains. The occurrence of $\pi$ - 


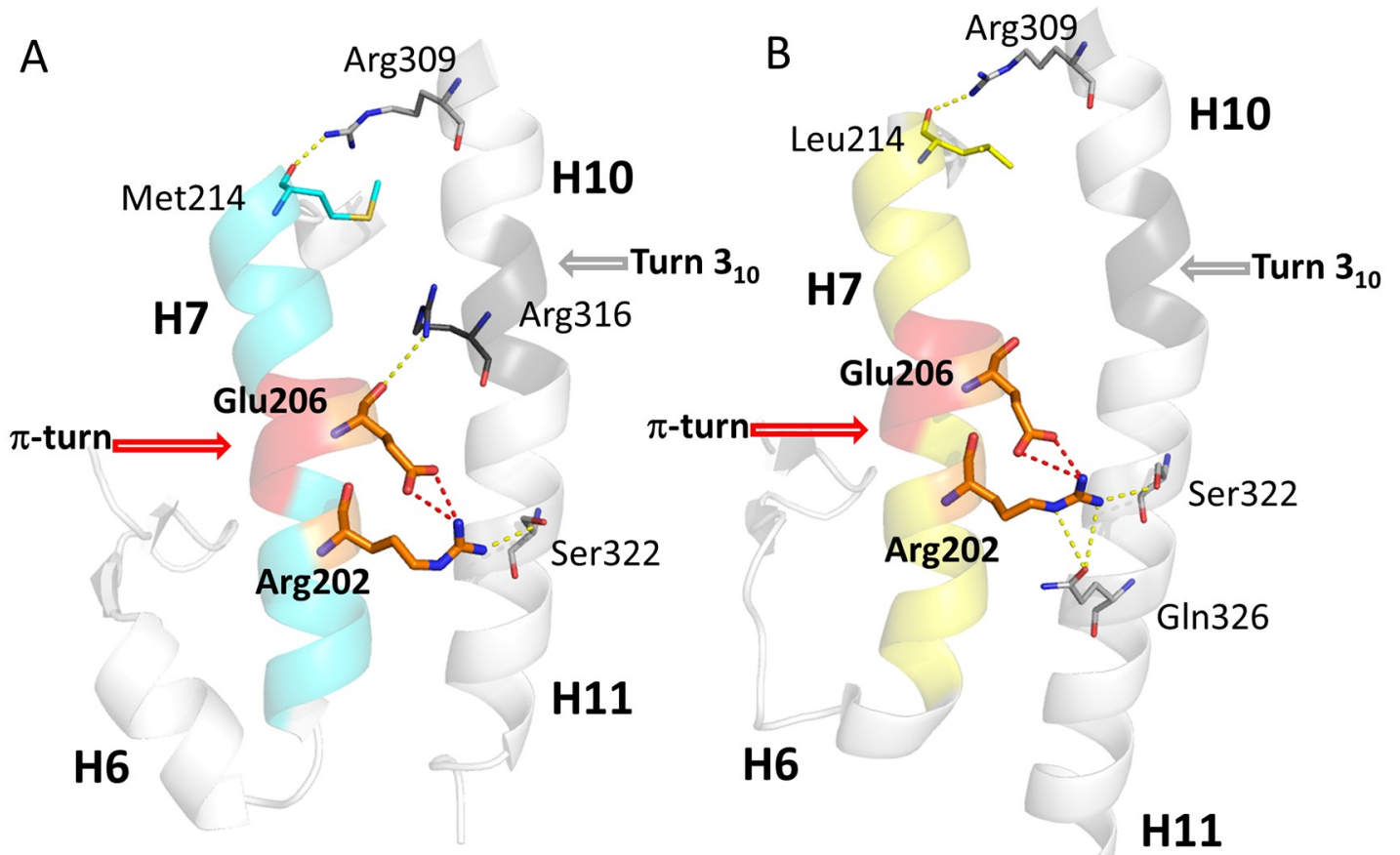

C

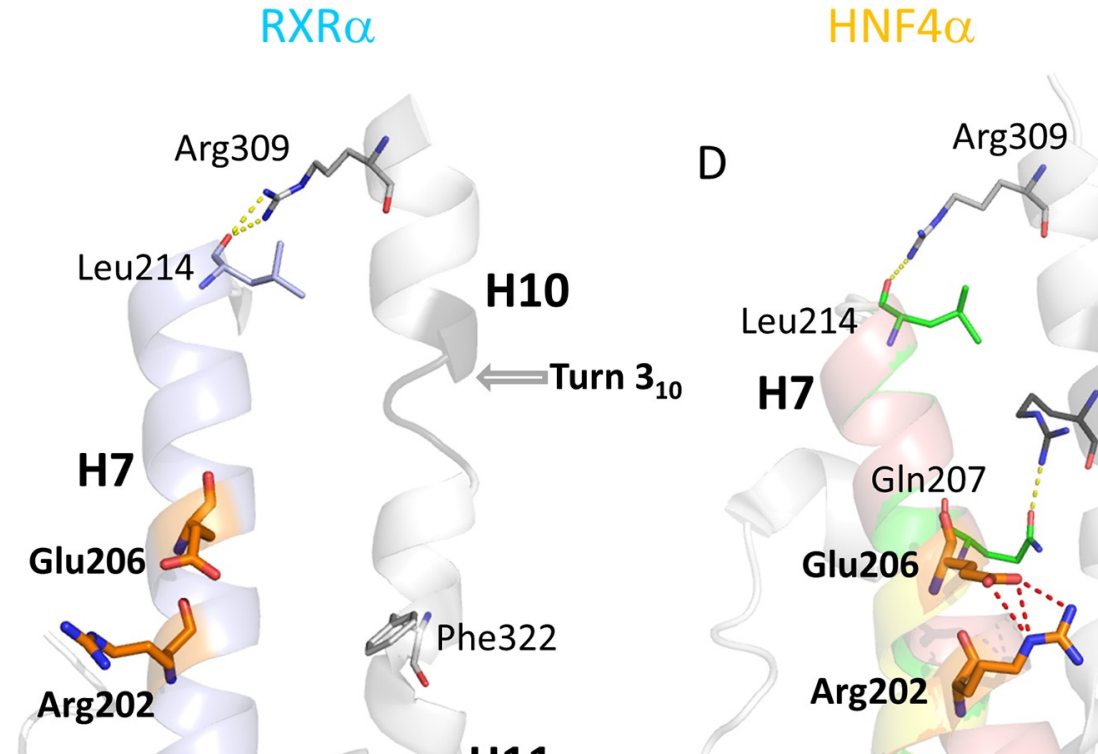

H10

$\mathrm{RXR} \alpha$

HNF4 $\alpha$

H11

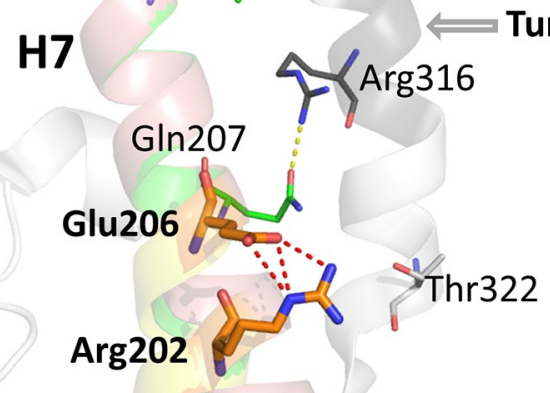

H11

H6

H6

PNR

COUP-TF II 
Fig 2. The environment of the $\pi$-turn in helix $\mathbf{H 7}$ of nuclear receptors. The environment of the $\pi$-turn is shown for (A) RXR $\alpha$, (B) HNF4 $\alpha$, (C) PNR and (D) COUP-TFII LBDs. The $\pi$-turn is shown in red, the $\pi$-turn motif residues (R202 and E206) are shown in orange. The $3_{10}$ helix at the $\mathrm{H} 10-\mathrm{H} 11$ junction in dark gray. In all cases, the $\mathrm{C}$-terminal part of helix $\mathrm{H} 7$ is anchored to the $\mathrm{N}$-terminal part of $\mathrm{H} 10$ by a conserved arginine (R309). Helix H7 is shown in blue and yellow ribbon representation for (A) RXR $\alpha$ and (B) $H N F 4 \alpha$. The side chains of the signature motif residues, R202 and E206, and S322 of H11 form a triad of H-bonds (A) and (B). R316 at the H10-H11 junction in (A) RXR $\alpha$ and Q326 in H11 of (B) HNF4 $\alpha$ complete the set of conserved bonds. For (C) PNR and (D) COUP-TFII, $\mathrm{H} 11$ is unstructured and the amino acid shift due to the absence of $\pi$-turn only affects the $\mathrm{N}$-terminus of $\mathrm{H} 7$, while no major changes occur at the C-terminal part, in line with the structural alignment of the corresponding residues. In (C), PNR is depicted with $\mathrm{H} 7$ in light violet. Contacts between $\mathrm{H} 7$ and $\mathrm{H} 10-\mathrm{H} 11$ are only observed at the C-terminus of $\mathrm{H} 7$. In helix H11, a phenylalanine residue (F322) replaces the serine residue S322 of RXR that is important for interaction with the $\pi$-turn. In (D), the original conformation of COUP-TFII is shown in green, the re-refined H7 conformers (see Materials and methods.) are shown in salmon and yellow for the straight and the curved helical conformations, respectively. A threonine residue (T322) in H11 replaces S322 of RXR H11 that interacts with the $\pi$-turn. The figures are based on the PDB structures $1 \mathrm{DKF}$ for (A) RXR $\alpha$, 4IQR for (B) HNF4 $\alpha, 4 \mathrm{LOG}$ for (C) PNR and on the PDB structure 3CJW and our re-refined structure for (D) COUP-TFII.

https://doi.org/10.1371/journal.pgen.1009492.g002

turns in the receptors that are considered to be at the origin of the NR family raises several questions, notably concerning the functional implications of this structural feature.

A conserved RxxxE motif, where the two invariant residues $\mathrm{R}$ and $\mathrm{E}$ form an intra-helical salt bridge further characterizes this specific conformation. In a $\pi$-helical loop, also called a $\pi$ turn, the $\mathrm{N}+4$ classical hydrogen bonds of the $\alpha$-helix are replaced by $\mathrm{N}+5$ hydrogen bonds $[25,26]$. The $\pi$-helical geometry results in the protrusion of the $\mathrm{E}$ residue out of the axis of the helix H7 with the two polar residues, E and R, closer to the helices H10-H11. Their side-chains form intricates inter- and intra-molecular interactions, stabilizing the per se energetically unfavorable $\pi$-helical conformation. The glutamate residue allows the formation of an intra-molecular salt-bridge with the conserved arginine residue of the motif. The arginine residue helps connect helix $\mathrm{H} 7$ to helices H10-H11 through binding to a conserved serine residue in helix H11 (S322 on the alignment, S427 in RXR $\alpha H S$, S1 Fig and Table 1). An additional hydrogenbond is observed between the $\pi$-turn and helices H10-H11. In RXR-USP, the H-bond is formed between E206 (E352 in hRXR $\alpha$ ) and R316 in H10 (R421 in hRXR $\alpha$ ) (Fig 2A). In HNF4, R202 (R267 in hHNF4 $\alpha$ ) binds to Q326 of H11 (Q350 in HNF4 $\alpha$ ) (Figs 2B and S1 and Table 1).

The $\pi$-bulge induced shift of residues only affects the $\mathrm{N}$-terminal part of $\mathrm{H} 7$. The C-terminal side is anchored by a conserved bond between the carbonyl group of residue M/L214 (H7) and the side chain of R309 (H10). A similar type of interaction pattern prevails for both receptors and leads to strong interactions between $\mathrm{H} 7$ and H10-H11. These helices, together with the loop H8-H9 and helix H9, are the main contributors to the canonical NR LBD

Table 1. Amino acid residue mapping for nuclear receptors considered in this study. $\mathrm{H}$ stands for helices. 'Alignment residue' is the generic numbering used in this study (S1 Fig). The class specific residues are specified by I and II for class I and class II NRs, respectively, with boxes colored in blue and green, respectively, or in cyan for residues common to class I and class II, together with the corresponding residue number [17]. The H7 column with yellow boxes refers to residues of the $\pi$-turn, further highlighting the differential conservation of the p-turn.

\begin{tabular}{|c|c|c|c|c|c|c|c|c|c|c|c|c|c|c|c|}
\hline \multirow{3}{*}{$\begin{array}{c}\text { Secondary structure } \\
\text { Class specific } \\
\text { Alignment residue }\end{array}$} & \multirow{3}{*}{$\begin{array}{c}\text { H4-H5 } \\
\text { I (W) } \\
109\end{array}$} & \multirow{3}{*}{$\begin{array}{c}\text { H5 } \\
\text { II }(\mathrm{E}, \mathrm{D}) \\
111 \\
\end{array}$} & \multicolumn{5}{|c|}{ H7 } & \multirow{3}{*}{$\begin{array}{c}\text { H8 } \\
\text { I; II (E) } \\
220\end{array}$} & \multicolumn{2}{|c|}{ loop H8-H9 } & \multicolumn{3}{|c|}{ H10 } & \multicolumn{2}{|c|}{ H11 } \\
\hline & & & \multicolumn{2}{|c|}{$\pi$-turn motif } & \multirow[b]{2}{*}{207} & \multirow[b]{2}{*}{210} & \multirow[b]{2}{*}{214} & & & II (R) & $\mathrm{I}(\mathrm{R})$ & & I; II (R) & & \\
\hline & & & 202 & 206 & & & & & 262 & 263 & 309 & 316 & 321 & 322 & 326 \\
\hline Brelivet numbering & 40 & 42 & - & - & - & - & - & 50 & 61 & 62 & 93 & 100 & 105 & 106 & - \\
\hline hRXRa & W305 & E307 & R348 & E352 & L353 & K356 & M360 & E366 & D379 & S380 & R414 & R421 & R426 & S427 & K431 \\
\hline hHNF4a & A 224 & E226 & R267 & E271 & L272 & P275 & L279 & E285 & D298 & A299 & R333 & L340 & Q345 & S346 & Q350 \\
\hline hCOUP-TFII & W249 & E251 & R293 & E297 & Q298 & K301 & L305 & E311 & D324 & A 325 & R359 & R366 & R371 & T372 & S376 \\
\hline hPNR & W257 & E259 & R301 & E305 & T306 & R309 & L313 & E319 & E332 & $\mathrm{T} 333$ & R367 & L374 & R379 & F380 & E384 \\
\hline hRAR $\alpha$ & C265 & D267 & D307 & A311 & F312 & Q315 & L319 & E325 & D338 & R339 & M373 & K380 & R385 & S386 & K390 \\
\hline hTR $\alpha$ & C309 & E311 & D351 & D355 & L356 & S359 & F363 & E369 & D382 & R383 & F417 & K424 & R429 & M430 & C434 \\
\hline
\end{tabular}

https://doi.org/10.1371/journal.pgen.1009492.t001 
dimerization interface. Another interesting observation is worth mentioning: in RXR, the contact between the main chain of E206 (H7, $\pi$-turn) and the side chain of R316 (H10) occurs in a place where the $\alpha$-helical conformation of H10 is locally changed to a short 3(10) helix characterized by $\mathrm{N}+3$ hydrogen bonds. This peculiar 3(10) conformation of $\mathrm{H} 10$ is observed for all known NRs structures, except for the pregnane $\mathrm{X}$ receptor (PXR) and the steroidogenic factor 1 (SF1) that have classical $\alpha$-helices (e.g. PXR, PDB: 1ILG, [27]; SF1,PDB: 4QJR, [28]). In order to correlate the presence of the RxxxE motif with the occurrence of a $\pi$-turn in $\mathrm{H} 7$, we carried out a structure-sequence analysis focused on $\mathrm{H} 7$ over several thousands of protein sequences. All available nuclear receptor sequences were taken into consideration. For 49 of them, at least one crystal structure was available. The RxxxE motif in $\mathrm{H} 7$ was found to be present in the NR2F group (COUP-TF, seven-up (SVP46/7-UP), V-erbA-related protein 2 (EAR2)) as well as in the Photoreceptor-specific nuclear receptor (PNR) belonging to the subfamily NR2E (but not in FAX, and the tailless receptors (TLL or TLX)). Whereas no crystal structure is available for SVP and EAR-2 LBD, crystal structures were reported for COUP-TFII (PDB: 3CJW, [29]) and PNR (PDB: 4LOG, [30]). None of these structures exhibits a $\pi$-turn conformation or a salt bridge between $\mathrm{R}$ and $\mathrm{E}$ residues of the RxxxE motif (Fig $2 \mathrm{C}$ and $2 \mathrm{D}$ ).

In PNR LBD, $\mathrm{H} 7$ exhibits a canonical $\alpha$-helical conformation with no visible distortions. No intra-molecular interactions are seen between residues $\mathrm{R}$ and $\mathrm{E}$ of the motif. The serine residue observed in RXR H11 (S322) that is important for the stability of the $\pi$-turn is replaced by F322 in PNR. This residue would generate a steric clash with a $\pi$-turn conformer. If a $\pi$-helix would be present in PNR, the offset induced by the bulge would change the position of E200 that would then point into the direction of $\mathrm{H} 5-\mathrm{H} 6$, more specifically into a hydrophobic region composed of several leucine residues that would not favor interaction.

The $\pi$-turn is also absent in the crystal structure of COUP-TFII [29]. The N-terminal part of $\mathrm{H} 7$ is partially disordered and lacks a stabilizing interaction with H11. Furthermore, the neighbouring helix $\mathrm{H} 6$ and the upstream connecting $\beta$-sheet are not present in the model. A closer inspection of the electron density map suggests that the $\mathrm{N}$-terminal part of $\mathrm{H} 7$ could adopt different conformations. Since this part of the protein is critical for our analysis of the RxxxE motif and the structural features associated to it, we refined the protein structure around this location by iterative building of residues in the non-interpreted electron density map followed by crystallographic refinements using PHENIX software (S2 Fig and S1 Table). The newly refined electron density map shows that helix $\mathrm{H} 7$ is more extended at its $\mathrm{N}$-terminal side and adopts two conformations, a regular straight $\alpha$-helix and a curved one bent at the level of the $\pi$-turn. The $\mathrm{C}$-terminal parts of the two helical conformations overlap nicely, while their N-terminal ends are $6 \AA$ apart. These two conformations are in equilibrium in the crystal, alternating between nearest neighbour molecules to ensure optimal packing and are likely to be natural conformations. The dynamics of $\mathrm{H7}$ resulting from the lack of stabilization through interactions with $\mathrm{H} 11$ promotes the adaptability to packing constraints with a subsequent disorder of this subdomain. The intra-helical salt bridge between the side chains of the conserved arginine R202 and glutamic acid E206 of the motif is conserved, but rotated to a position where no interaction between the motif and H10-H11 can take place, since the shift induced by the absence of the $\pi$-turn prevents E206 from binding R316. Instead the connection is made with its neighboring residue Q207 (Q298 in hCOUP-TFII). A threonine residue that does not interact with $\mathrm{H} 7$ residues replaces the conserved serine residue in $\mathrm{H} 11$ that stabilizes the $\pi$-turn in RXR-USP and HNF4. In addition, no interactions are seen between $\mathrm{H} 7$ and H5-H6. Altogether, our analysis of the re-refined crystallographic structure of COUP-TFII unambiguously demonstrates that the RxxxE motif present in the sequence of this receptor is structurally associated neither with a $\pi$-turn in helix $\mathrm{H} 7$, nor with a 3(10) helical turn as suggested in the original structure [29]. 


\section{The $\pi$-turn motif is ancestral and has been lost several times independently}

The analysis of sponge nuclear receptor sequences show the presence of the RxxxE motif in helix H7 of SpNR1, but not of SpNR2. SpNR1 is associated with the group of nuclear receptors NR2B, C, D, E, F as well as NR3/4/5/6 subfamilies, while SpNR2 belongs to the HNF4-like subfamily. The markers of dimerization for class I and class II corroborate this interpretation (E5, W40, K/R55, R/K93, R105 for class I NRs; E/D42, R62, H/R/K90 for class II NRs and E50 and R105 universally conserved) [17]. Indeed, SpNR1 encompasses all of the class I markers, while in SpNR2, two class I markers (W40 and R105) are missing. Interestingly, the same class markers are absent in HNF4. Homology modelling of SpNR1 using a reference panel of nuclear receptor structures, which in majority do not have a $\pi$-turn, indicates the presence of a $\pi$-turn in $98 \%$ of the generated models (see Materials and methods). Furthermore, when SpNR1 replaces RXR in the structures of homodimers or heterodimers, the essential dimeric interactions are conserved. This suggests that the essential distinguishing features of RXR that can exist as a homodimer as well as a heterodimerization partner were already present in SpNR1.

In order to understand the evolutionary dynamics of the $\pi$-turn motif conservation, we plotted the presence of the $\pi$-turn motif, as well as that of the RxxxE motif on a phylogenetic tree of NR sequences (Figs 3A and S3). Our tree topology is fully consistent with previous studies $[8,9]$. The tree allows to robustly position most NR subfamilies, even though a major unresolved trichotomy still subsists concerning the branching of the NR3 and NR5/6 families relative to the robust NR7/NR4/NR1 cluster. Interestingly, our current sampling regarding sponges and other early metazoans sequences indicates that, within SpNR1, a lineage-specific amplification has occurred in calcareous sponges, leading to four distinct paralogues (numbered P1 to P4 in S3 Fig). Our analysis therefore includes the whole currently known diversity of early NRs (see S3 Fig).

Taken together, these data suggest that the $\pi$-turn, and its associated RxxxE motif were present ancestrally in the primordial nuclear receptors and lost in several rapidly evolving lineages of basal NRs (e.g. sponge SpNR2, some paralogous sponge SpNR1), as well as in the major derived NR subfamilies (i.e. NR2EF, NR3, NR1 etc.).

\section{The ancestral NR activated transcription as a homodimer}

Nuclear receptors exhibit three different modes of oligomerization: homodimer binding (e.g. steroid receptors or HNF4), heterodimers with the promiscuous partner RXR (e.g. TR, RAR, LXR or PPAR) and monomer binding (e.g. SF1 or Rev-erb) [1,31] (Fig 1B). It is important to note that these modes of binding are not mutually exclusive. For example, homodimer formation has been demonstrated for Rev-erb which can also bind to DNA as a monomer [32]. Similarly, RXR can form either homodimers or heterodimers. This oligomeric behavior is related to the mode of binding to DNA, since response elements are derivatives of a canonical sequence (A/GGGTCA) that can be modified, extended or duplicated therefore offering a large palette of possible NR-selective binding modes [31]. As mentioned above, the final oligomeric status is thus the result of the interplay between the strong dimerization interface in the LBD and a weaker one in the DBD which is crucial for response element selection [1]. To trace back the evolutionary history of the dimerization abilities of NRs, it is therefore necessary to fully disentangle the DNA binding and response element selection from the oligomeric status. For this reason, we focused our analysis on the major dimerization interface of the isolated LBD.

To understand the evolution of dimerization of nuclear receptors, we mapped the dimerization patterns of each receptor, using the four states already defined [33-36] on a simplified version of our updated phylogeny, which is fully consistent in its topology with previous 
A

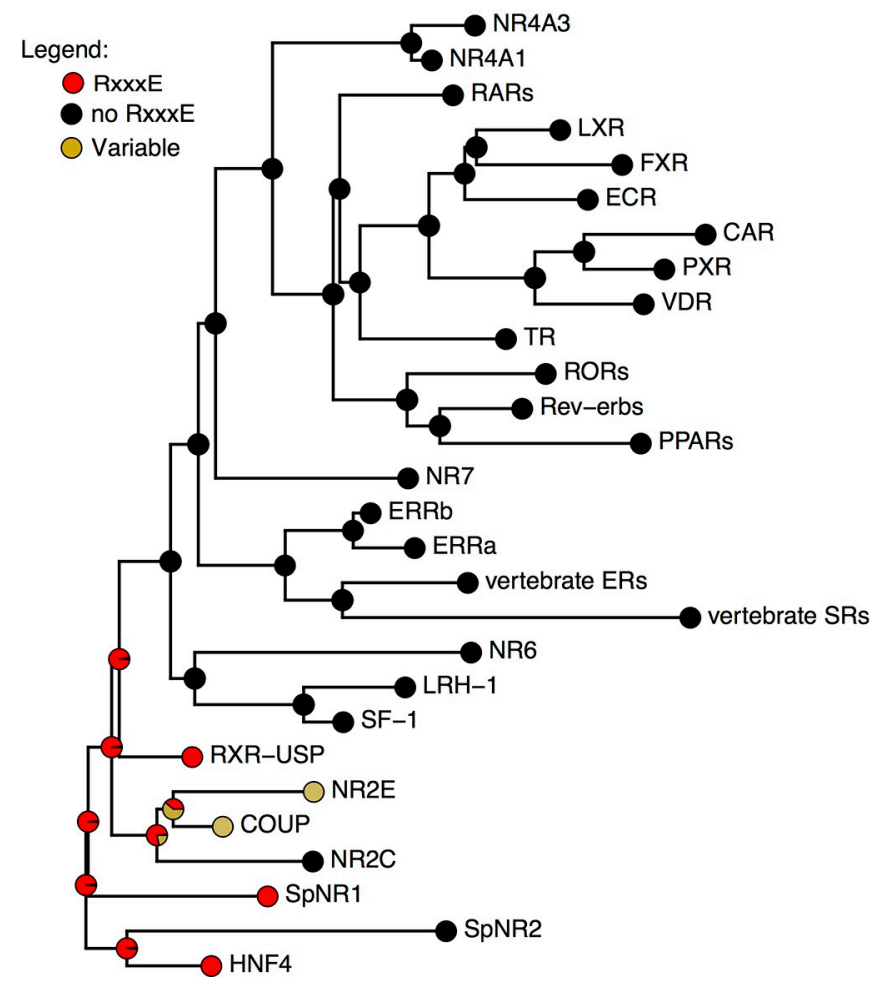

B

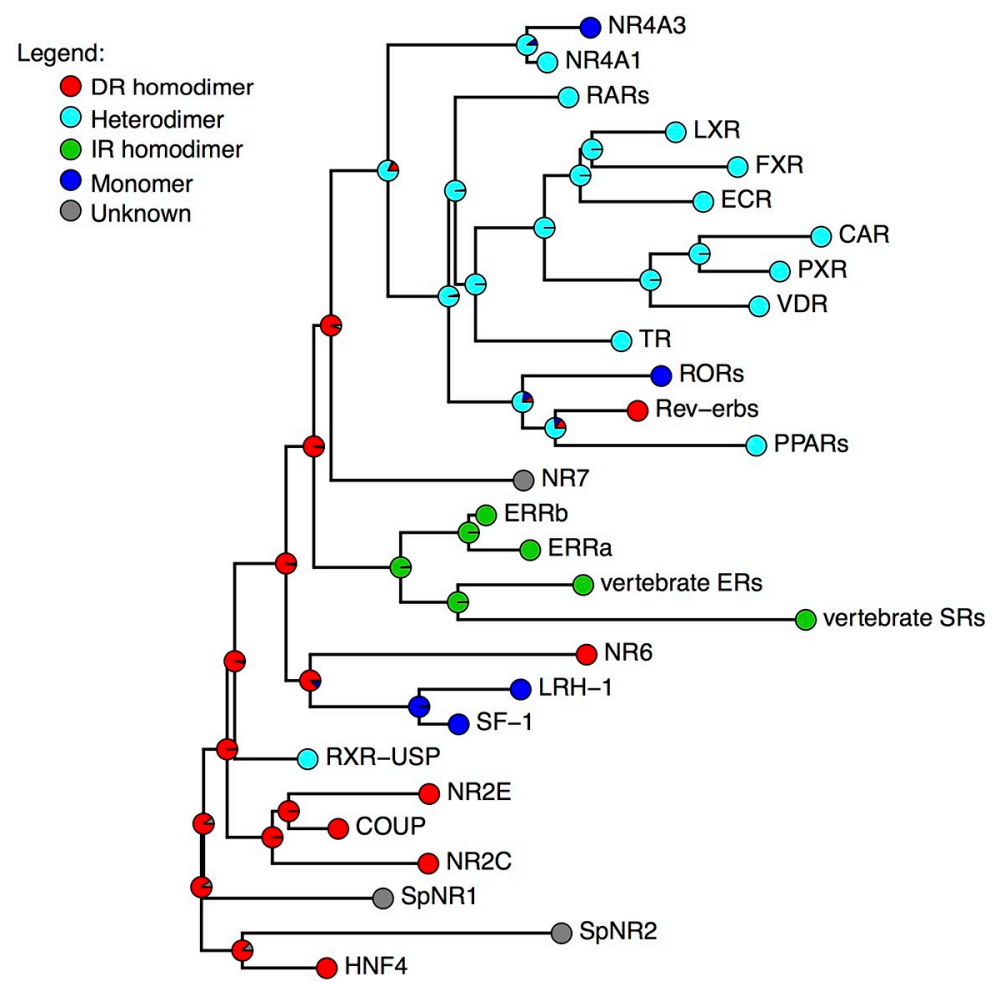

Fig 3. Evolution of the RxxxE motives compared to the dimerization modes during the history of the NR family. A. Evolution of RxxxE motives, as reconstructed using ancestral mapping. B. Evolution of dimerization modes. Source files and script are provided in dryad under the following doi:10.5061/dryad.kkwh70s48.

https://doi.org/10.1371/journal.pgen.1009492.g003

publications based on a similar dataset [8,9]. We adopted a conservative strategy in that when no experimental data was available, we coded the relevant oligomerization ability as unknown even if clear class I or class II residues can safely indicate the dimerization mode [17].

The ancestral state reconstruction for every node of the phylogeny illustrates successive complexification of the binding mode. Ancestrally, the binding mode is that of a homodimer, then only a heterodimer binding mode emerged once at the basis of the NR1 and NR4 families, while the monomer binding mode appeared several times independently from either from DR-homodimer or from RXR-heterodimers (Fig 3B).

\section{The $\pi$-turn residues are required for HNF4 biological function}

According to the NR partition into class I (monomers and homodimers) and class II (heterodimers) NRs, RXR-USP and HNF4 belong to the class I. Class I differentially conserved residues (i.e. residues strictly conserved in class I and strictly absent in class II NRs) define a class-specific interaction pattern that connects together $\mathrm{H} 1$ to $\mathrm{H} 8$ and $\mathrm{H} 8$ to $\mathrm{H} 10$, thereby networking the ligand binding pocket to the dimerization interface [17]. Examination of the class I-conserved residues in HNF4 indicates that this receptor is an outlier of the class I NRs. In fact, two class I invariant residues, W109 (W40 in the alignment given by [17]) and R321 (R105) are not conserved for HNF4, W109 being replaced by an alanine residue and R321 by a glutamine residue (Table 1). In HNF4, the residues A109 and Q321 are essentially conserved from cnidarians to mammals. In contrast, in sponge spNR2, W109 is replaced by a valine residue and R321 is mainly replaced by lysine or tyrosine residues but in a context lacking the $\pi$-turn. Note that these two residues have important structural and functional roles. W109 is located at the 
junction of H4-H5, a highly conserved structural feature of the class I NR family and an interaction hot spot for ligands. It was shown to be involved in a ligand-dependent allosteric mechanism in RXR [37]. The arginine residue R321 in H10 is an important residue of the dimerization interface highly conserved for all NRs, except in HNF4 and the oxosteroids subgroup (the androgen (AR), glucocorticoid (GR), mineralocorticoid (MR) and progesterone (PR) receptors). In the latter family, and only there, this mutation is associated with the mutation of the residue E111 (E42) that is normally strictly conserved in the whole NR family. In RXR, but not in HNF4, this amino acid residue binds to R321 and contributes to the stability of the homodimer. Altogether, the mutation of the two highly conserved residues W109 and R321 in HNF4 highlight the early divergence of this receptor from the rest of the family.

The analysis of the crystal structures of the HNF $4 \alpha$ homodimer shows that a large contribution to the stability of the homodimer comes from the unusual stacking of the tryptophan residue W325 (W349 in hHNF4 $\alpha$ ) in H10 with the corresponding residue of the other subunit (Fig 4A). These residues and the corresponding contacts they form are specific to this receptor family. Furthermore, several other residues of $\mathrm{H} 10$ belonging to one subunit contact helix H9 and the loop H8-H9 of the other subunit, thus forming a strong interaction network. In addition, the $\pi$-turn residue E206 in one subunit interacts with the region H10-H11, thereby forming intermolecular stacking interactions with D262 (D298 in hHNF4 $\alpha$ ) in the loop H8-H9 of the other subunit (Fig 4A). In summary, we observe an intricate and unusual interaction network that involve residues of the $\pi$-turn as well as helices $\mathrm{H} 9$ and $\mathrm{H} 10$ in both subunits. When we compared HNF4 to RXR, the numerous contacts (H-bonds, VdW...) that link together the two LBD subunits result in a larger buried surface at the dimer interface, consistent with an energetically more stable oligomer.

The functional importance of the $\pi$-turn of HNF4 to the homodimerization process was demonstrated in earlier studies, where the $\pi$-turn residues R202 and E206 were mutated and the functional consequence assessed [38]. In this work, it was shown that removing the charges of R202 and E206 impairs dimerization of the protein in solution and affect the HNF4 $\alpha$ transcriptional activity in a variety of different cell lines. The impairment on transcriptional activity is even larger for the deletion mutant $\Delta \mathrm{E} 206$ (E262 in hHNF4 $\alpha$ ), which was also shown to be less efficient in recruiting transcriptional partners, such as SRC-1 and PGC-1. To correlate with biological effects, we searched the library of human HNF4 mutations reported for MODY1 syndrome and for various cancers that feature HNF4 somatic mutations. We found a small number of somatic mutations in the $\pi$-turn motif, especially affecting R202 (R267 in hHNF4 $\alpha$ ), suggesting that this residue is indeed important for the biology of HNF4 in humans (HGMD database [39]).

Two specific features could explain absence of HNF4 heterodimers. First, the numerous $\mathrm{H}$ bonds linking $\mathrm{H} 9$ and $\mathrm{H} 10$ of the LBD partners, mostly absent in RXR, are largely responsible for the strength of HNF4 homodimers, and much more stable than RXR ones (Fig 4A and 4B). Interestingly in RXR heterodimers, the number of bonds between helix H10 of RXR and helix H9 of the partner NR increases significantly (Fig 4C). Second, the mutation of a class I and II marker, R381 in RXR, Q321 in HNF4, is another remarkable feature. In RXR homo and heterodimers, R321 is bound to serine 322, conserved in most class II partners.

\section{The $\pi$-turn motif is critical for RXR homodimer formation}

In contrast to the strong and intricate homodimerization interaction interface of HNF4, the dimerization interface of RXR dimer involves less contacts, as shown in Fig 4B. The scarcity of the interactions between the two subunits of the homodimer suggests a less stable dimer. Few interactions are observed between $\mathrm{H} 10$ of one subunit and $\mathrm{H} 9-\mathrm{H} 10$ and the loop $\mathrm{H} 8-\mathrm{H} 9$ of the 
A

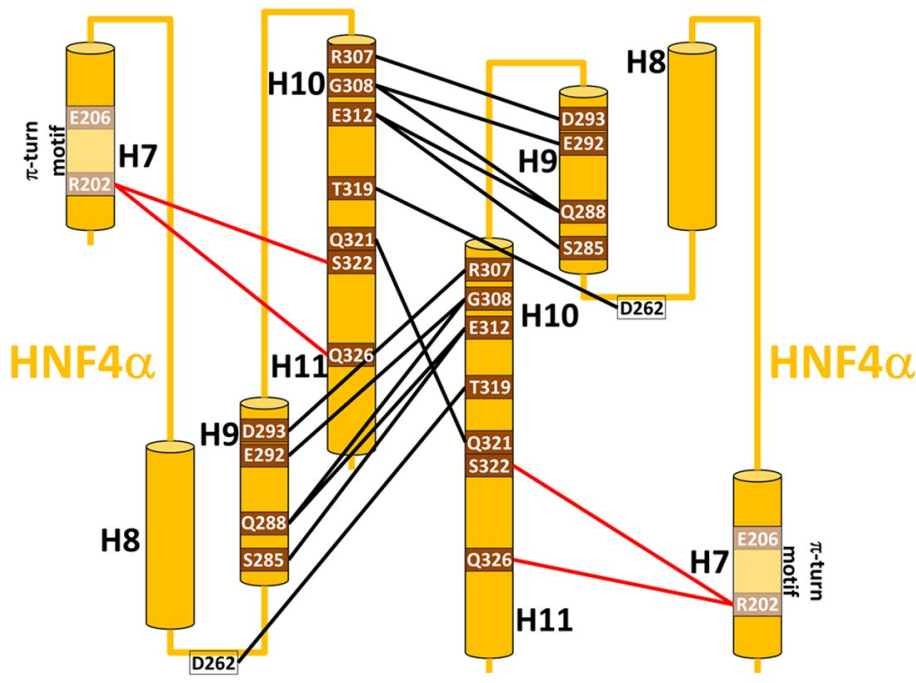

C

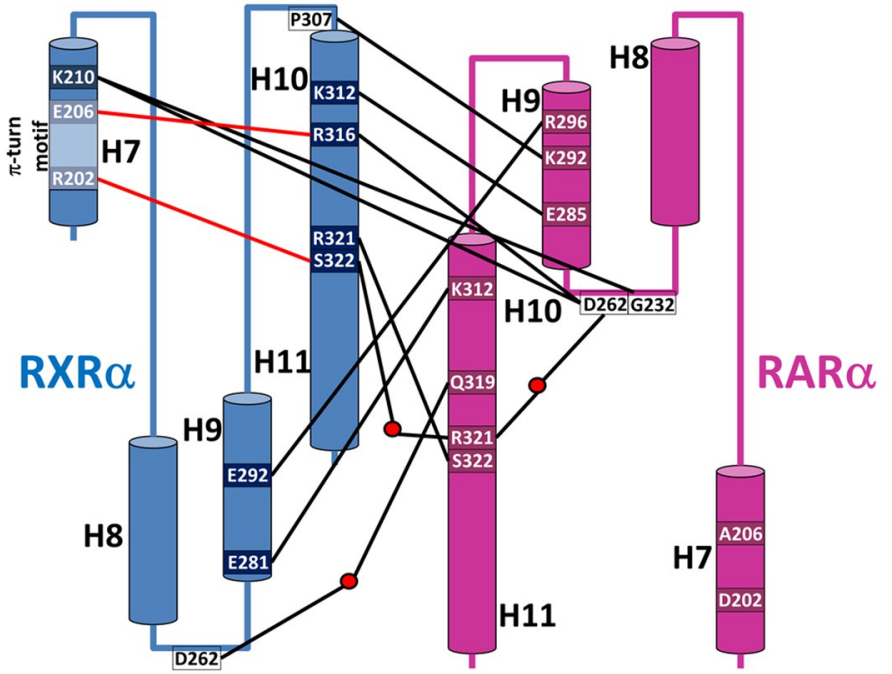

B

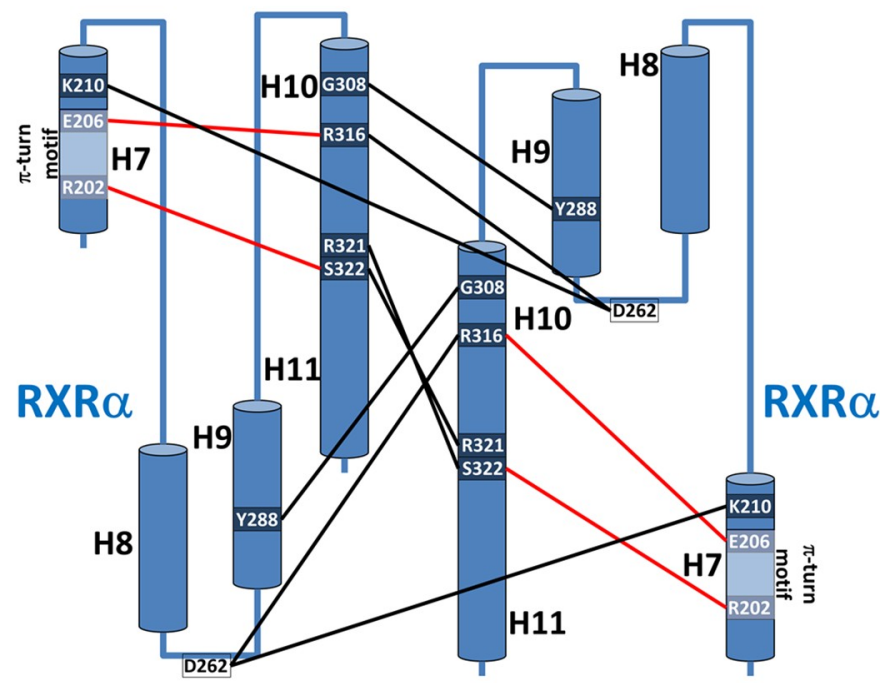

D

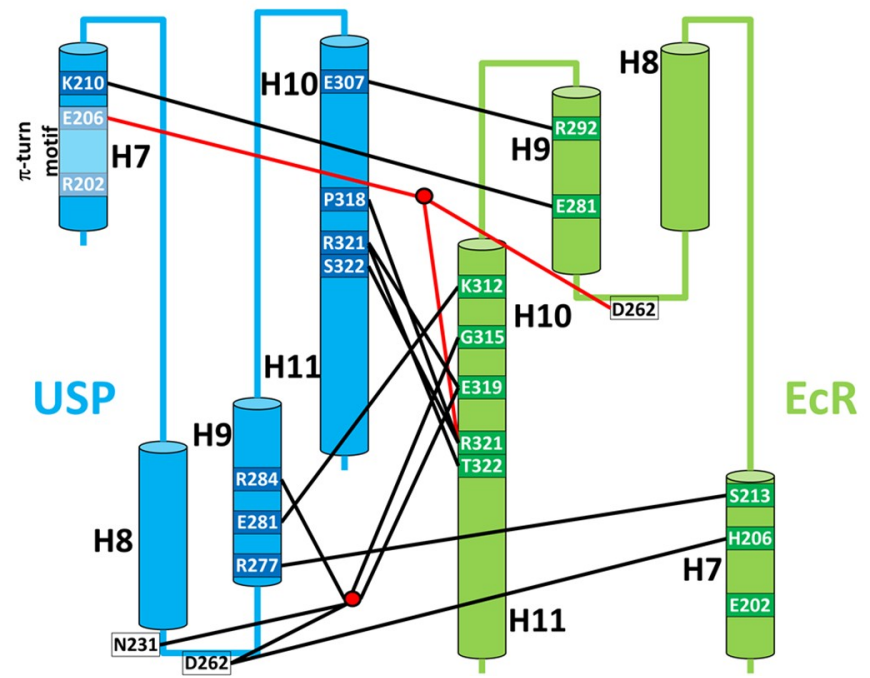

Fig 4. Schematic representation of the dimer stabilizing bonding interactions. The set of $\alpha$-helices $\mathrm{H} 7$ to $\mathrm{H} 11$ of each subunit of the LBD dimer is represented by cylinders. Water molecules are shown by red dots. Dimerization bonds between the two LBD subunits are represented by black lines and bonds between residues R202 and E206 of the $\pi$-turn motif and residues at the dimer interface are shown by red lines. Shown in the scheme are bonds which are most frequently observed in the majority of the available structures. (A) HNF4 $\alpha$ LBD. The dimer is symmetric and mainly stabilized by cross-contacts between helices H9 and H10. The $\pi$-turn contributes in an indirect manner to the dimerization interface through internal bonds with residues of helix H11. (B) RXR LBD. The dimerization is symmetrical, but less stabilizing bonds can be observed as compared to the HNF4 case. Interactions are also more uniformly distributed over the entire interface, but helix H9 is weakly involved in the dimerization interface, whereas the $\pi$-turn is directly and strongly implicated. (C) RXR-RAR. The dimer is asymmetrical, and helices H9 are more strongly involved in the dimerization interface. The helix $\mathrm{H} 7$ of RXR directly contributes to the dimer interface through its $\pi$-turn. There is no contribution of RAR helix H7. (D.) USP-EcR. The interface is asymmetrical, but less asymmetric than for the different heterodimers formed with RXR. Note the unusual role played by helix $\mathrm{H} 7$ of EcR in the dimerization process [48].

https://doi.org/10.1371/journal.pgen.1009492.g004

other subunit. Importantly, the $\pi$-turn residues play a crucial role in the stability of the homodimer. R202 and E206 are both involved in the dimerization interface. R202 link together S322 (H11) in the same subunit to R321 in H10 of the other subunit. E206 links together R316 (H10) found in the same subunit to D262 located in the loop L8-9 of the other subunit. The latter residue further interacts with $\mathrm{K} 210$ located at the C-ter of the $\pi$-turn. Altogether, the 
structural analysis shows that the $\pi$-turn residues are strongly involved in the homodimerization interface.

To assess the functional importance of the $\pi$-turn residues for RXR homodimerization, we sought the effects of mutating the critical residues of the $\pi$-turn motif on the dimerization behavior of RXR. To address this question, we chose to mutate E206 (E352 in hRXR $\alpha$ ) of the RXXXE motif of RXR $\alpha$ LBD either into an alanine residue or to delete it completely from the LBD protein construct and relied on biophysical methods, including analytical size-exclusion chromatography (SEC), analytical ultracentrifugation (AUC) and native electrospray ionization mass spectrometry (ESI-MS) for the analysis of the oligomeric status of the wild-type and mutant proteins. In addition, we carried out molecular dynamics simulations of wild-type and mutant receptors to gain insights into the stability of the dimeric species.

The analytical SEC analysis was carried out using a S200 10/300 Superdex column by injecting the different proteins after the affinity purification step. The corresponding chromatograms, shown in S4A Fig, reveal notable differences between wild-type (wt) RXR $\alpha$ LBD and $\mathrm{E} 352 \mathrm{~A}$ and $\triangle \mathrm{E} 352$ mutant constructs. The three proteins have a peak in common at an elution volume that roughly corresponds to the exclusion volume of the column (called void in S4A Fig), and therefore to large oligomeric protein species. Two additional peaks are observed for wtRXR $\alpha$ LBD (called peak1 wt and peak2 wt in S4A Fig and indicated with red and blue symbols, respectively), whereas only one additional is seen for the mutants RXR $\alpha$ LBD peak (called peak mut in S4A Fig, and indicated with cyan and grey symbols for E352A RXR $\alpha$ and $\Delta \mathrm{E} 352$ $\mathrm{RXR} \alpha$, respectively), with a similar elution volume. This indicates that the two mutant LBD constructs behave differently compared to wtRXR LBD and lack the larger species that compose peak 1 of wtRXR LBD. Since all SEC peaks correspond to pure protein samples, as shown in the SDS-PAGE gel in the insert of S4A Fig, the difference in the size of the protein species composing each peak can solely be attributed to different protein oligomerization states and not to any co-purified contaminant species.

To further identify the SEC-separated species, SEC was online coupled to native ESI-MS for accurate oligomeric state assessment [40]. SEC-native MS analysis of wtRXR $\alpha$ LBD reveals two peaks, as shown in Fig 5. The first peak (shown in red in the inset of Fig 5) consists of tetramers, whereas the second peak (shown in blue in the inset of Fig 5 ) is composed of dimers and monomers (Fig 5 and S2 Table). In contrast, the main peak of both RXR mutants corresponds to dimeric and monomeric species only, while no tetramers are detected (Fig 5 and S2 Table). Of note, under strictly identical experimental and instrumental conditions, the $\Delta \mathrm{E} 352$ RXR $\alpha$ mutant exhibits more dimers than the E352A RXR $\alpha$ mutant, which might suggest a slightly increased homodimer stability for $\triangle \mathrm{E} 352$. Altogether, the MS analysis indicates that mutating E352 of the RxxxE motif of RXR $\alpha$ LBD dramatically impairs noncovalent tetramer formation when compared to the wtRXR $\alpha$ LBD. However, we still observe a low abundance population of mutant RXR $\alpha$ LBD dimers in a large crowd of monomers. As strictly identical SEC columns could not be used off line and in-line with native MS, we further collected SEC peaks obtained on a S200 10/300 Superdex and analyzed the fractions by native ESI-MS (S5AS5D Fig and S3 Table). Again, native MS data analysis indicates that the wtRXR $\alpha$ LBD sample is composed of noncovalent tetramers and monomers (only low intensity dimers are detected) (S5A and S5B Fig and S3 Table), whereas mutant RXR $\alpha$ LBD samples do not exhibit any tetramer species, but rather a mixture of monomeric and dimeric populations (see S5C and S5D Fig and S3 Table).

We further analyzed the RXR SEC fractions by analytical ultracentrifugation (AUC). The AUC data are summarized in S4B Fig that shows the differential sedimentation coefficient distribution $\mathrm{c}(\mathrm{S})$ as a function of the sedimentation coefficient $\mathrm{S}$. Two $\mathrm{c}(\mathrm{S})$ peaks are observed for the sample corresponding to SEC peak 1 of wtRXR $\alpha$ LBD shown in red in S4A Fig and only 


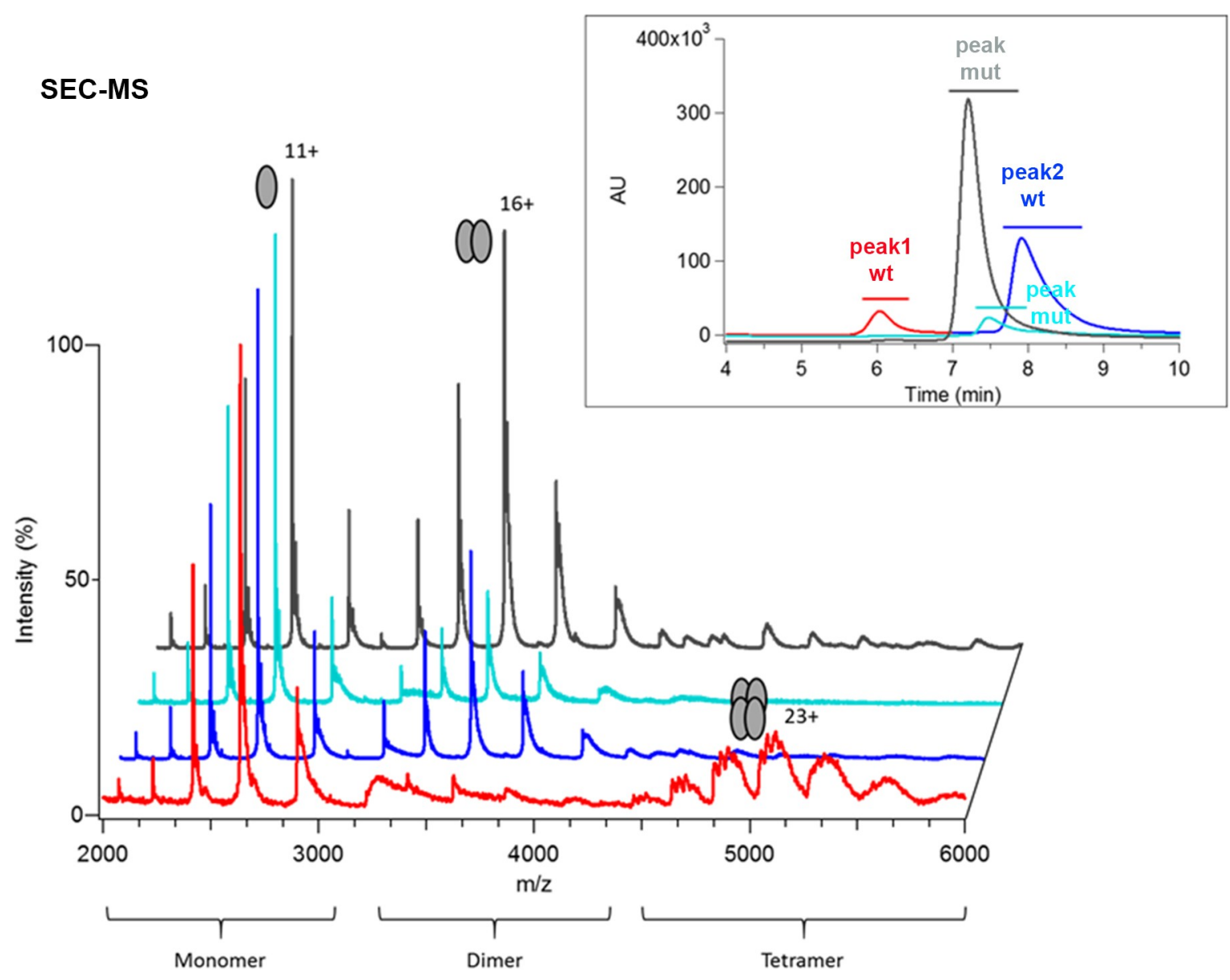

Fig 5. Oligomeric status of wild-type (wt) RXR $\alpha$ LDD and mutants, where the conserved Glu residue of the $\pi$-turn motif is mutated to Ala (E352A) or deleted ( $\triangle$ E352). Size-exclusion chromatography (SEC)-coupled native mass spectrometric analysis for wt RXR $\alpha$ LBD (red for the first peak and blue for the second peak), E352A RXR $\alpha$ LBD (cyan) and $\Delta \mathrm{E} 352 \mathrm{RXR} \alpha \mathrm{LBD}$ (grey). The insert depicts the sizeexclusion chromatograms of wt RXR $\alpha$ LBD and of mutants RXR $\alpha$ LBD (with the same color code). The region of the SEC peak considered for the integration of the mass spectra is shown as a line above the SEC peak. For wt RXR $\alpha$ LBD the tetrameric species is seen at the beginning (I) of the SEC chromatogram. In contrast, monomeric species is observed for all SEC peaks together with dimeric species. For mutant RXR $\alpha$ LBD species, no tetramer is observed in the SEC chromatogram and in the corresponding mass spectra where mainly monomeric and dimeric species are observed.

https://doi.org/10.1371/journal.pgen.1009492.g005

one peak for the other samples (shown in blue, cyan and grey in S4A Fig). Detailed examination of the sedimentation data shows that for wtRXR $\alpha$ LBD, the SEC peak 1 is a heterogeneous sample with several species in dynamic equilibrium, including a majority of tetramers and smaller species down to the monomer, whereas the SEC peak 2 is composed of a mix of monomers and dimers. In the case of the RXR mutants, the AUC data analysis indicates that the samples consist mostly of monomers and a slight amount of dimers. Thus, the two peaks observed in the differential sedimentation coefficient distribution $\mathrm{c}(\mathrm{S})$ correspond to the tetrameric species for large $S$ value and essentially to monomer species for the SEC peak 2 of $w t R X R \alpha$ LBD. For the mutants, monomeric species prevail, but the larger width of the $c(S)$ peak suggests the formation of rapidly associating/dissociating dimers from the larger monomer pool. Importantly, no tetramer is observed for the mutants RXR $\alpha$ LBD E352A and $\triangle \mathrm{E} 352$. The AUC results show that monomers and tetramers are the main species of wtRXR $\alpha \mathrm{LBD}$, in full consistency with SEC-native MS observations (Figs 5 and S5A-S5D) native gel electrophoresis (S5E Fig). Note that a unique band is observed in the native gel of the mutant RXR $\alpha$ LBD 
species which might be attributed to the rapidly exchanging monomer/dimer species or to the dominant dimeric fraction, as observed in the AUC and MS analyses.

Altogether, the biophysical data indicates that noncovalent tetramer formation is impaired for the RXR $\alpha$ LBD E352 mutants, in stark contrast with the wild-type protein. The RXR $\alpha$ tetramer is composed of a non-covalently bound dimer of RXR $\alpha$ homodimer and importantly represents the main reservoir of RXR $\alpha$ homodimer available in the cell, as shown in vivo and in vitro [41,42]. It was shown that disruption of the tetramerization interface of RXR $\alpha$ by mutating conserved phenylalanine residues in helix H11 (depicted in S5F Fig) results in transcriptionally defective protein, without affecting the overall fold of the protein, nor ligand binding, dimer formation or DNA binding. Here, strikingly, we show that the mutation or the deletion of E352 in $\mathrm{H} 7$ impairs tetramer formation, by destabilizing the mutant RXR $\alpha$ homodimer species. This residue is far from the tetramerization interface composed of helices $\mathrm{H} 3, \mathrm{H} 11$ and H12 (S4F Fig) [43,44]. However, residues of the $\pi$-turn interact with helix H11 and help stabilize its conformation. The mutation of the conserved Glu residue of the $\pi$-turn does not prevent homodimer formation since the interface also encompasses other mostly conserved and hydrophobic residues [45]. However, it is likely to destabilize helix H11 and as a consequence to weaken the homodimer interface, enough to lead to the destabilization of the tetramerization interface, as observed experimentally.

We finally carried out Molecular Dynamics (MD) simulations to investigate whether the propensity for dimerization within RXR is affected. MD simulations of $50 \mathrm{~ns}$ were thus performed starting from a 1.9 ̊́ resolution crystal structure of HsRXR LBD (PDB: 1MVC [46]. Three sequences were used, including the E357A and $\triangle \mathrm{E} 357$ mutants. The total binding free energies were then calculated for each complex. The results, shown in $\$ 4$ Table, suggest that wt $\mathrm{RXR} \alpha \mathrm{LBD}$ is the most stable homodimeric complex, followed by $\triangle \mathrm{E} 352 \mathrm{RXR} \alpha \mathrm{LBD}$ and E352A RXR $\alpha$ LBD. Examination of the resulting structures after the MD simulations shows that for the E352 mutants, the contacts between $\mathrm{H} 7$ and $\mathrm{H} 10$ of one subunit and the loop H8-H9 and H9 of the other subunit are dramatically weakened (S6A-S6D Fig). Altogether, the biophysical characterization and MD simulations suggest that the E352 deletion and mutation has a destabilizing action of RXR homodimeric association, hampering tetramer formation for both mutants.

\section{The $\pi$-turn allowed RXR to evolve as a promiscuous partner for heterodimerization}

In contrast to $\mathrm{HNF} 4 \alpha, \mathrm{RXR} \alpha$ can form heterodimers with NR partners. In all the cases, the heterodimerization interface is always asymmetric, whereby helix $\mathrm{H} 7$ of RXR is closer to the loop H8-H9 of the partner than the reverse. An intricate network of interactions spans the entire interface between RXR and its NR partner that involve helices H7, H8 and $\mathrm{H} 9$ and the loop H8-H9. The observation is consistent with experimental data indicating that the heterodimers are more stable than the RXR $\alpha$ homodimer [47]. The asymmetry of the dimerization interface has a direct impact on the number and the type of interactions. By taking RXR $\alpha /$ RAR $\alpha$ as an example (PDB: 1DKF [48]), we observe a scarce number of interactions between the loop H8-H9 of RXR $\alpha$ and helix H7 of RAR $\alpha$, while numerous interactions are seen in the reverse situation, i.e. between the loop $\mathrm{H} 8-\mathrm{H} 9$ of RAR $\alpha$ and $\mathrm{H} 7$ of $\mathrm{RXR} \alpha$, and in particular its $\pi$-turn residues R202, T205, E206 and K210 (Fig 4C). The direct or water-mediated interactions between the latter residues and residues of the loop H8-H9 of RAR $\alpha$, D262, Q264 and $\mathrm{D} 265$, are made possible by the mere presence of the $\pi$-turn in $\mathrm{RXR} \alpha$, whereas the absence of $\pi$-turn in the partner LBD prevents the establishment of most bonds. Thus, the generation of the strong asymmetry in the dimerization interface is highly dependent on the presence of the 
$\pi$-turn in RXR and its concomitant absence in partner NRs. The asymmetry of the heterodimer together with the involvement of class conserved residues of the RXR partner in the heterodimer interactions likely favored RXR as a common dimerization partner and led to the emergence of class II NRs as partners of RXR.

Finally, from the evolutionary point of view, it is interesting to consider the well-known ecdysone receptor, which is found in insects and other arthropods, in particular in insects and which is made of a heterodimer between EcR and USP, the ortholog of RXR. Several crystal structures of EcR/USP-RXR LBDs are available from different insect species [20,49-51]. All of the structures exhibit an asymmetric heterodimeric interface, just like vertebrate NRs, with a similar interaction pattern as seen in the previous example of RAR $\alpha / \mathrm{RXR} \alpha$. In particular, helix $\mathrm{H} 7$ of USP-RXR that encompasses the $\pi$-turn makes direct and water-mediated interaction with the loop H8-H9 of EcR (Figs 4D and S7A). On the other hand, analyses of the structures indicate that, depending on the insect species considered, contacts between the helix $\mathrm{H} 7 \mathrm{of} \mathrm{EcR}$ and USP-RXR may vary enormously [20,49-51]. In the more basal insect species, such as the beetle Tribolium castaneum $(\mathrm{Tc})$ (Coleoptera) and the silverleaf whitefly Bemisia tabaci (Bt) (Hemiptera), no or few contacts are observed (none for Bt and one bond between $\mathrm{H} 441 \mathrm{in} \mathrm{H} 7$ from EcR to Asp 325 in the loop H8-H9 of USP). In more recent species, such as the moth Heliothis virescens $(\mathrm{Hv})$ (Lepidoptera), in stark contrast, numerous bonds are observed linking the helix $\mathrm{H} 7 \mathrm{of} \mathrm{EcR}$ and the loop H8-H9 and the helix H9 of USP-RXR (S7A Fig). The origin of this difference between species comes from the position of the loop USP-RXR H8-H9 which is close enough for interaction with $\mathrm{EcR}$ in $\mathrm{Hv}$, but not in Tc and Bt (S7B Fig). This discrepancy reflects more profound differences in the overall structure of USP-RXR among the different species $[49,52]$. In fact, USP-RXR of the basal insect species are more similar to the mammalian RXR than to sequences of USP-RXR of more recent species that encompass the Lepidoptera (moths and butterflies) and Diptera (flies, mosquitos) groups. Therefore, the peculiarity observed for HvEcR/HvUSP-RXR merely reflects the high evolutionary divergence of Lepidoptera and Diptera compared to the other clades [52-54]. However, the analysis of the more recent species EcR/ USP-RXR LBDs suggests that independently of the existing interactions made between $\mathrm{H} 7$ of EcR and USP-RXR, the asymmetry of the dimerization interface that is dependent of the presence of the $\pi$-turn in USP-RXR remains a conserved feature. Altogether, the analyses of the EcR/ USP-RXR structures nicely illustrate the evolutionary conservation of the heterodimerization interface, its asymmetry and the involvement of the $\pi$-turn into the dimerization mechanism.

To substantiate our hypothesis for the role played by the $\pi$-turn in the heterodimerization, we experimentally characterized the heterodimers between RXR $\alpha$ LBD and PPAR $\alpha$ LBD for the wild-type and the E352 RXR $\alpha$ mutants. We used SEC-coupled to native MS to relatively quantify the heterodimeric PPAR $\alpha / R X R \alpha$ population in the complex mixture between RXR $\alpha$ (wt or mutant) and its partner PPAR $\alpha$ LBD. Fig 6 summarizes the relative abundances of monomeric and heterodimeric species as deduced from native MS results (S8 Fig). All RXR $\alpha$ construct (wt but more interestingly also E352 mutants) allow formation of PPAR $\alpha / R X R \alpha$ heterodimers (Figs 6 and S8, together with the presence of monomeric RXR $\alpha$ and PPAR $\alpha$. However, there is a strong reduction in the relative PPAR $\alpha / R X R \alpha$ heterodimer population between PPAR $\alpha /$ wtRXR $\alpha$ and PPAR $\alpha / E 352$ mutants (Figs 6 and S8), along with increased amounts of free monomeric RXR $\alpha$ detected.

\section{Discussion}

\section{The $\pi$-turn clusters a crucial interaction network in basal NRs}

In this paper, we focused on the $\pi$-turn motif which is present in nuclear receptors located at the base of the NR evolutionary tree. The structural importance of this motif that clusters a 

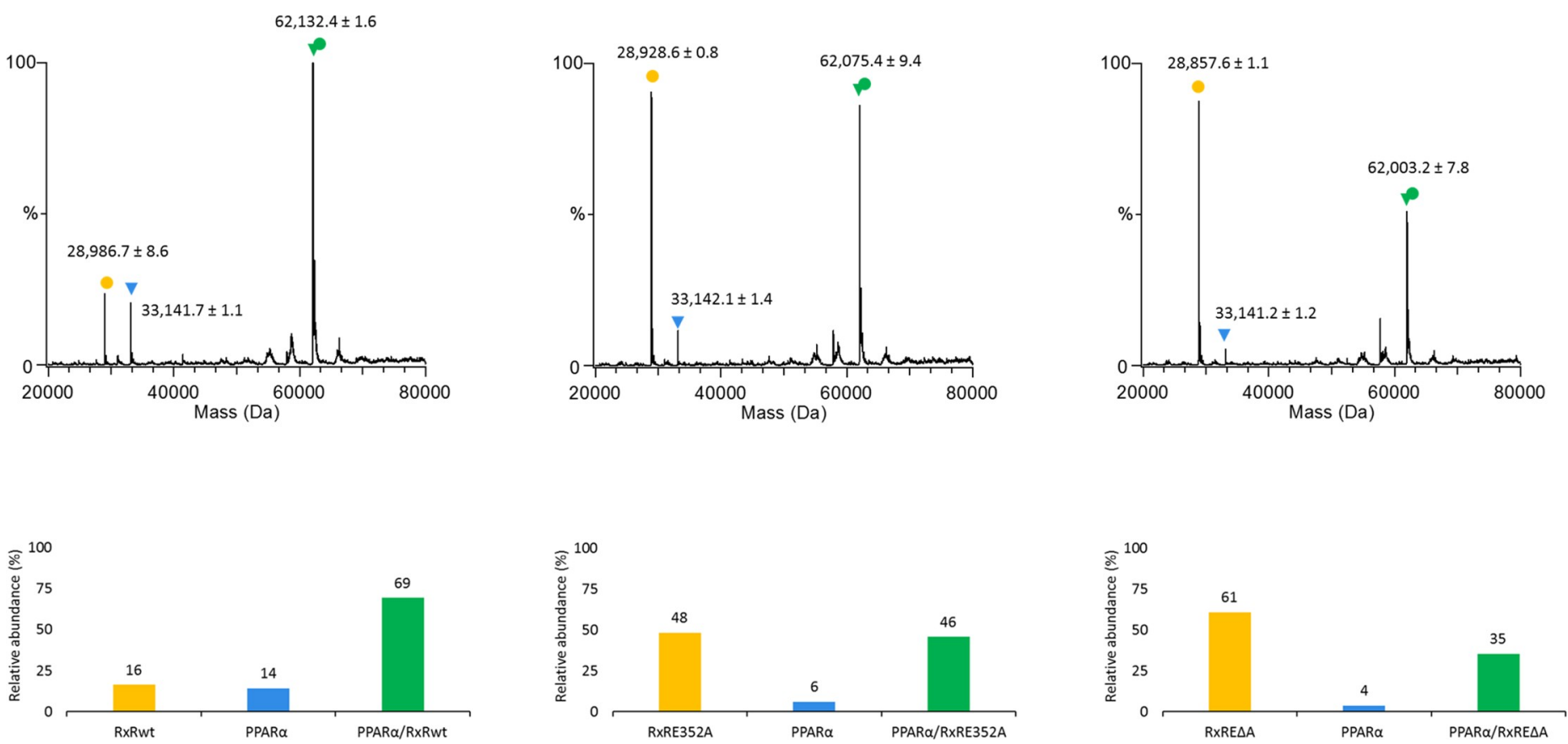

Fig 6. Heterodimerization capacity of wild-type (wt) RXR $\alpha$ LBD and mutant E352A and $\triangle E 352$ RXR LBD with PPARalpha. Size-exclusion chromatography (SEC)-coupled native mass spectrometry (MS) analysis for complex mixture of PPAR $\alpha$ LBD with either (A) wt RXR $\alpha$ LBD, (B) E352A RXR $\alpha$ LBD or (C) $\Delta$ E352 RXR $\alpha$ LBD. The isolated RXR $\alpha$ LBD (wt or mutants) is depicted in yellow, isolated PPAR $\alpha$ LBD in blue and heterodimeric PPAR $\alpha / R X R \alpha$ LBDs in green. Mass spectra obtained by deconvolution of the raw data (shown in S6 Fig) for the three different complex mixtures are shown, together with the quantification of the species from the SEC-native MS analysis of the PPAR $\alpha / R X R \alpha$ LBD complex mixture, shown below in the form of histograms.

network of amino acid residue interactions, its complex evolutionary history and conservation in basal NRs strongly suggest that it is a key structural element for the functional diversification of NRs. Our structural analysis reveals that the $\pi$-turn, located within helix $\mathrm{H} 7$, is always associated with the presence of an RxxxE motif. Furthermore, our 3D homology modeling study allowed us to infer the presence of a $\pi$-turn in NRs that harbor the RxxxE sequence motif, but for which no structural and functional data are available. As a result, we observed the presence of a $\pi$-turn in HNF4 of bilaterians and basal metazoans, such as cnidarians and placozoans (Trichoplax), as well as in RXRs of bilaterians and cnidarians and in SpNR1. The latter case is particularly interesting, since SpNR1 together with SpNR2 represent the only NRs found in sponges. These two receptors, which are used to root the NR superfamily tree, are considered to be the most basal NRs and thus define the two major subdivisions in the NR evolutionary tree, one containing SpNR2 and HNF4 (NR2A) and the other one containing SpNR1 and all the other NRs (Fig 3A) [9].

Our phylogenetic analysis enabled us to hypothesize that the $\pi$-turn is an ancestral motif that was present early in the primordial NRs and that was further differentially lost at least five times during the NR evolution. Due to the similarities between the $\pi$-turn present in the structures of RXR and those of HNF4, we strongly support the " $\pi$-turn early" scenario, rather than the alternative " $\pi$-turn late" scenario of the late independent origin of the $\pi$-turn in HNF4s, RXRs and SpNR1 (Fig 3A). Based on our analysis, we inferred that a $\pi$-turn similar to those seen in HNF4 and RXR should be present in SpNR1. A crystallographic study of SpNR1 LBD would be the ideal test for our hypothesis.

The structure-sequence analysis of NRs that exhibit a RxxxE motif indicates that among all NRs whose structure is known, only RXR-USP and HNF4 possess a peculiar $\pi$-helical 
geometry. This intrinsically unstable $\pi$-helical conformation requires strong stabilizing interactions between the $\mathrm{N}$ - and the $\mathrm{C}$-terminal parts of $\mathrm{H} 7$ and neighboring regions in its molecular environment to hold together this topological feature. Importantly, the $\pi$-turn of $\mathrm{H} 7$ thus gives rise to specific intricate interactions with helices $\mathrm{H} 10$ and $\mathrm{H} 11$, both being crucial element of the dimerization interface. As a matter of fact, the junction between the two helices H10-H11 encompasses a 3(10) conformation and a specific leucine rich sequence (LLLXXL or LLXXL) at the N-terminal part of H10. These structural features, which induce a kink in the region of helices $\mathrm{H} 10-\mathrm{H} 11$, make possible crucial and complementary interactions between the $\pi$-turn conformer of $\mathrm{H} 7$ and $\mathrm{H} 11$, notably between the arginine residue of the RxxE motif and the serine residue of $\mathrm{H} 11$. Therefore, the $\pi$-turn is at the heart of the network of interactions present in RXR and HNF4 from the origin for the stabilization of the LBD and for the formation of a stable homodimerization interface (Fig 4). These interactions allowed the ancestral receptor to bind DNA response elements as a dimer in a cooperative manner, an ability that increased the DNA binding site selectivity. It is important however to emphasize that the $\pi$-turn is not necessary for the homodimerization of all nuclear receptors, but only for RXR and HNF4. In fact, steroid NRs, such as the estrogen receptor (ER) and the estrogenrelated receptor (ERR) homodimerize in the absence of $\pi$-turn and RxxxE motif, making use of the same secondary structural elements for building the dimerization interface as RXR and HNF4. From an evolutionary point of view, ER and ERR evolved in a way such as they underwent compensatory mutations leading to the disappearance of the RxxxE motif, but conserving most of the other interacting residues. On the other hand, the later evolved oxosteroid nuclear receptors (AR, GR, MR and PR) are different in their dimerization properties. Their ligand binding domain does not dimerize in the same manner as ER and ERR. In fact, there is a marked sequence difference of the residues at the interface compared to the whole nuclear receptor family and the presence of an additional conserved region at the C-terminal end of the LBD that hampers the oxosteroid receptors to dimerize in a classical way $[1,55]$ that still needs to be uncovered.

In protein structures, $\pi$-helices and $\pi$-bulges are often associated with a specific function, making them powerful markers of protein evolution [23]. An accepted hypothesis about the emergence of $\pi$-bulges is their frequent implication as ligand binding site contributors such as in GPCRs (van der Kant and Vrient, 2014). In the case of NRs, a direct association with ligand binding is rather unlikely. For example, both apo and holo crystal structures are available for RXR and, importantly, show no significant differences in the $\pi$-turn environment. Note that the in vivo relevance of RXR ligands, such as 9-cis retinoic acid or DHA, is a highly debated and controversial issue [56]. Similarly, whereas all known HNF4 crystal structures are liganded, the biological significance of HNF4 ligands is not clear, since the latter are either non-exchangeable molecules found in the LBD structure or do not induce any transcriptional activity [57]. For SpNR1 and SpNR2, barely no information is available. Functional characterization of sponge receptors combined with phylogeny analysis and ancestral sequence reconstruction allowed Bridgham et al. to propose that NRs evolved from a ligand-activated ancestral receptor that existed near the base of the Metazoa, with fatty acids as possible ancestral ligands [9]. Taken together, these data indicate that the presence of the $\pi$-turn in RXR and HNF4 is not related to the ligand binding capability.

Interestingly, we observe that the absence of a $\pi$-turn is correlated with the loss of $\mathrm{H} 7-\mathrm{H} 11$ stabilizing bonds and as a consequence, is linked to a greater flexibility of the ligand binding site which is indeed partly composed of helices $\mathrm{H} 7$ and $\mathrm{H} 11$ [58]. Several examples of NR LBDs have been reported where the ligand binding pocket nicely adapts and molds to different types of ligands, by exhibiting remarkable changes in the structural elements composing the binding cavity. It is in particular the case for EcR [59], ER [60], VDR [61,62], PXR [63], and 
many other NRs. For all of them, the adaptation of the pocket to the ligand occurs through substantial changes of the region encompassing helices $\mathrm{H} 7$ and $\mathrm{H} 11$, and the $\beta$-sheet. Focusing on helix H7, the structural adaptation of this helix can occur only when it is devoid of the structural constraints that would be imposed by the presence of a $\pi$-turn. In other words, molding and adaptability to various ligand molecules is correlated to the absence of a $\pi$-turn. Thus, from the evolutionary point of view, the disappearance of the $\pi$-turn in more recent nuclear receptors (from NR1 and NR4 subfamilies) facilitated the binding of a variety of molecules and promoted their diversification.

The presence of a $\pi$-turn in RXR and HNF4 suggests that its maintenance is linked to a different function, namely dimerization (Fig 7). Our analysis supports the key role of the $\pi$-turn of RXR in heterodimer formation through numerous interactions with the loop H8-H9 and the helices $\mathrm{H} 9$ and $\mathrm{H} 10$ of the partner NR. Experimental evidence provided here for the case of PPAR $\alpha / R X R \alpha$ LBD fully supports our hypothesis. The lack of $\pi$-turn in the partner receptor strengthens the resulting asymmetric heterodimer. We hypothesize that the presence of the $\pi$ turn in RXR is a necessary condition for this receptor to be the ubiquitous dimerization partner of many different NRs. This structural feature is namely linked to a stiffening of the LBD structure, especially the heterodimerization region, allowing RXR to dimerize in a similar fashion with different partner receptors.

\section{The $\pi$-turn represents an unusual exaptation}

In protein science, it has always been thought that the $\pi$-turn is a structural feature that evolved in a way such as to accommodate novel functionalities. This is not the case here, since the $\pi$ turn is an ancestral motif that was instead lost during NR diversification. However, its presence or its absence is linked to critical biological functions. On the one hand, the presence of the $\pi$ turn in the most ancestral receptors is crucial for the stabilization of a homodimer interface in the context of small molecule binding in the LBD for sensor function. On the other hand, the loss of the $\pi$-turn in all subsequent NRs allowed their binding site to adapt to a different type of ligands and for a large group of them facilitated their heterodimerization with RXR in a stronger and asymmetric manner.

The origins of novelties still remain a central question in evolutionary biology. A fundamental question is how organisms constrained by natural selection can divert from existing schemes to set up novel structures or pathways. Among all possible strategies addressing this issue, pre-adaptations [64], which are also called exaptations, are one of the most important strategies. According to Gould and Vrba, exaptations are "features that now enhance fitness but were not built by natural selection for their current role" [65]. Numerous examples of exaptation have been proposed at the morphological and the molecular levels. Feathers used for bird flight originate indeed as thermoregulation devices in dinosaurs (they were also colored: reproductive and camouflage functions as well). At the molecular level, crystalline lenses first emerged as metabolic enzymes that were later on recruited in the eye for their light-refracting function [66]. More recently, cases of exaptation were identified in the case of retroviral envelope proteins that were recruited as placental proteins in early mammals [67] or in amphibians as a mechanism for functional reinforcement of a pheromone system [68]. Exaptations are furthermore frequently observed at the gene expression level, mainly through the recruitment of new gene regulatory elements allowing cooptation of gene function in novel organs, tissues or process. This is for example the case of the reinforcement of the courtship pheromone system in frogs via the co-option of the persuasin gene [68]. This has also been observed in several cases after gene duplication [69]. Exaptations are however less frequent for protein structures. One example are the bifunctional metabolic enzymes [70] or the sea urchin fibropellin protein, for 


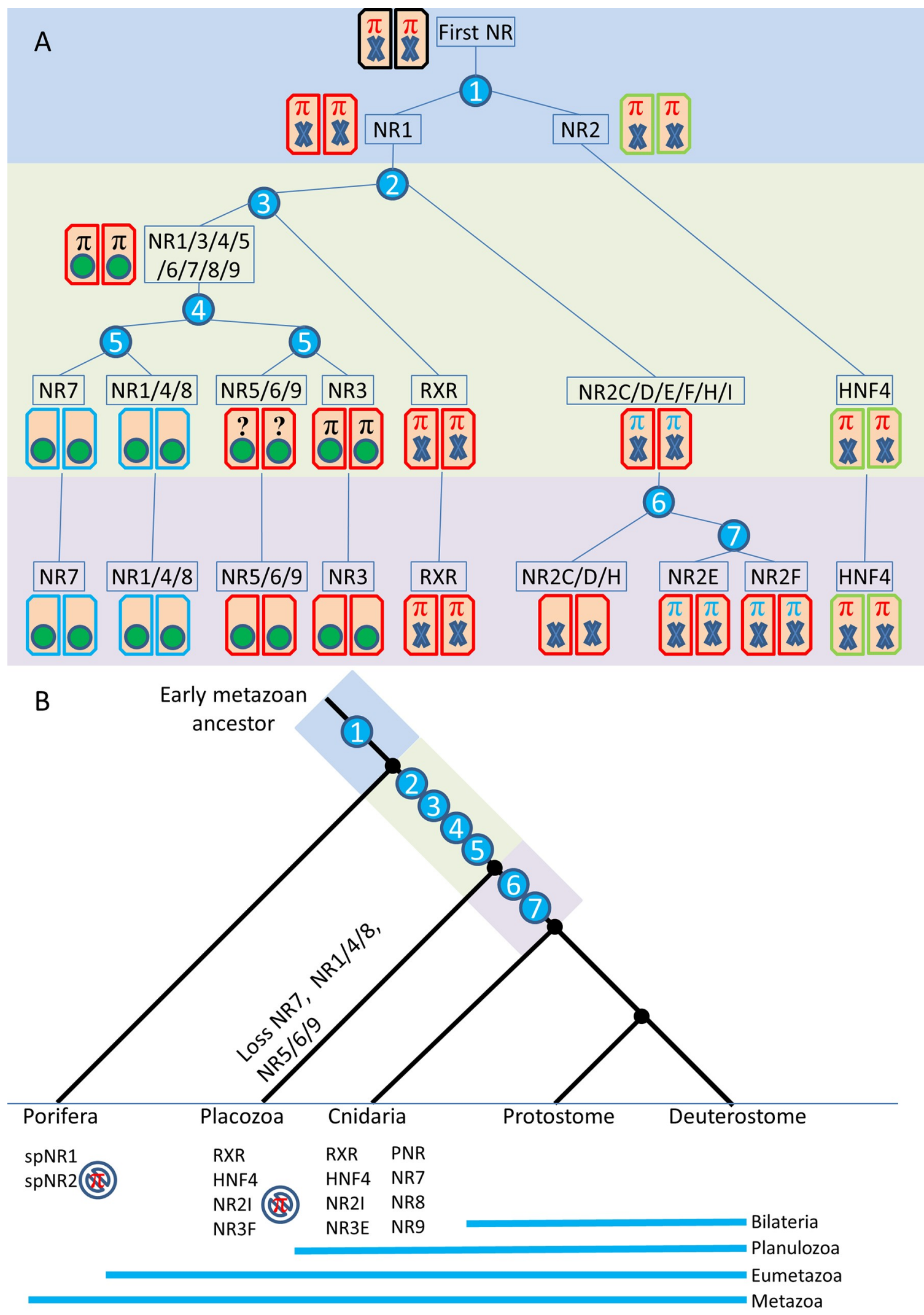


Fig 7. Evolution of the $\pi$-turn motif and dimerization interface. (A) Evolutionary history of the main receptor families in regard to $\pi$ turn and dimerization. The rectangles correspond to LBD monomers and their color indicates their class marker composition: red (e.g. RXR) for all Class I markers, green (e.g. HNF4) when the W109 and R321 are missing, and blue (e.g. NR7) for other Class I marker anomalies. The first NR is indicated in black as no information is available. The blue numbered circles are duplication events that are plotted on the tree on panel B. The ligand binding pocket is indicated as a green circle when it is liganded and a blue cross when there is no or weak non-specific interaction. The $\pi$-turn motif is indicated by a big PI symbol $(\pi)$, red for motif and structure present, black for motif present but no information about structure, blue for motif present and structure absent and '?' symbol when no information is available. (B) Phylogenetic position of the evolutionary events described in (A) (blue numbered circles). For porifera, placozoans and cnidarians, the minimal sets of receptors present in their last common ancestor are indicated.

https://doi.org/10.1371/journal.pgen.1009492.g007

which a dimerization motif evolved from a biotin binding domain [71]. Here, we propose that the $\pi$-turn, an ancestral structural feature that was present in ancient receptors, in particular in RXR and HNF4, was important for homodimerization and later utilized by RXR as a key structural element for heterodimerization. However, its loss allowed the reinforcement of ligand adaptation in other NRs. Both contrasting aspects eventually led to a substantial expansion of the repertoire of NR regulatory abilities.

To summarize, both the presence (in RXR) and the absence (in NR partners) of the $\pi$-turn led to the emergence of new NR function, namely the heterodimerization of RXR with partner receptors, leading to greater target site selection and the emergence of high affinity receptors due to more flexible binding site that could diversify in terms of ligand binding possibilities. We propose that the $\pi$-turn in NRs represents a case of structural exaptation, namely a trait whose benefit for the system is unrelated to the reason of its origination, but which allowed an unprecedented increase of the NR regulatory repertoire.

\section{Materials and methods}

\section{Structure refinement of COUP-TF LBD}

A careful analysis of the crystal structure of COUP-TFII and its corresponding electron density map reveald that large portions of the electron density in the region of $\mathrm{H} 7$ could not be interpreted. The main problems are located between $\mathrm{H} 5$ and $\mathrm{H} 7$, with no visible electronic density for the $\beta$-sheet and $\mathrm{H} 6$ connecting $\mathrm{H} 5$ to a disordered $\mathrm{N}$-terminal part of $\mathrm{H} 7$. For the latter a closer inspection to the electron density map suggests that the N-terminal part of $\mathrm{H} 7$ could adopt different conformations. Since this region was critical for our analysis of the RxxxE motif and the structural features associated to it, we decided to further improve the protein structure around this location by iterative building in Coot of residues in the non-interpreted electron density map followed by a crystallographic refinement using Phenix. This work resulted in better crystallographic quality factors $\mathrm{R}$ and Rfree and to a more confident interpretation of the electron density map (see S1 Table).

After crystallographic re-refinement, we observe that in the crystal packing helix $\mathrm{H} 7 \mathrm{can}$ adopt two helical structures, together with a lengthening of helix $\mathrm{H} 7$ at its $\mathrm{N}$-terminal side as compared to the original helix of the PDB structure (S2 Fig). The two novel conformations correspond to a regular straight and a curved $\alpha$-helix bent at the level of the putative $\pi$-turn. The C-terminal parts of the two helices overlap nicely, while their $\mathrm{N}$-terminal ends are located over $6 \AA$ apart. These conformations are in equilibrium in the crystal, alternating between nearest neighbour molecules to ensure optimal packing and are likely to be natural conformations. The dynamics of $\mathrm{H} 7$ resulting from the absence of a stabilizing $\mathrm{H} 11$ promotes the adaptability to packing constraints with a subsequent disorder of this subdomain. In fact, the lengthening of the original single helix $\mathrm{H} 7$ to the size of the re-refined one would lead to steric clashes between crystallographic dimers. 
The second important observation is the absence of the $\pi$-turn. Although the electron density was not clear enough to confidently build the side chain of R293, some density can be seen that could correspond to the guanidinium group of the arginine in the straight conformation of the helix, indicating that in this conformation, the intra-helical salt bridge between the side chains of the arginine and the glutamic acid of the RxxxE motif could be maintained. However, this intra-helical salt bridge is rotated to a position such that no interaction between the $\mathrm{H} 7$ motif and H10-H11 can take place. The shift induced by the absence of the $\pi$-turn prevents E206 from binding R316, instead the connection is made with its neighboring residue Q207 (Q298 in hCOUP-TFII). The conserved serine residue of RXR H11 that stabilizes the $\pi$-turn in RXR-USP and HNF4 is replaced by a threonine residue, but without interacting with $\mathrm{H} 7$ residues. Furthermore, no interactions are seen between $\mathrm{H} 7$ and H5-H6. Of note, helix H7 after refinement does not exhibit a 3(10) helical turns as suggested in the original structure.

\section{Evolutionary analysis}

Collected NR sequences were aligned using Clustal Omega (Sievers and Higgins, 2014) and alignments were checked manually and edited with Seaview (Gouy et al., 2010). Phylogenetic trees were built using PHYML (Guindon and Gascuel, 2003). Following model testing using AIC and BIC criteria as implemented in the SMS software (Lefort at al., 2017), we selected the LG model (Le and Gascuel, 2010) with a gamma law and estimation of the proportion of invariable sites. The reliability of nodes was assessed by likelihood-ratio test (Anisimova and Gascuel, 2006). Ancestral character reconstruction and stochastic mapping (Huelsenbeck et al., 2003) were performed under R version 3.2.2 (R Core Team, 2015) using the make.simmap function as implemented in the phytools package version 0.5.0. (Revell, 2012). Character evolution was inferred using a model of symmetrical transition rates between the character states (SYM). 10000 character histories were sampled to allow the incorporation of the uncertainty associated with the transition between different states. Inferred state frequencies for ancestral nodes were plotted using the describe.simmap function. Commands and sources files for ancestral mapping are provided in the Dryad repository under the following doi:10. 5061/dryad.kkwh70s48.

\section{Cloning, expression and purification for biophysical studies}

HsRXR $\alpha$ LBD, wild type (T223-T468) and mutants E352A and $\triangle E 352$, were cloned into the pET15b expression vector. HsPPAR $\alpha$ LBD (I195-Y468) was cloned in a pET15b expression vector. Each individual vector was transformed into Escherichia coli BL21 (DE3), grown at $37^{\circ} \mathrm{C}$ and induced for protein expression at an $\mathrm{OD}_{600 \mathrm{~nm}}$ of 0.6 with $1 \mathrm{mM} \mathrm{IPTG}$ at $25^{\circ} \mathrm{C}$ for 3 hours. The corresponding cell pellet was resuspended in binding buffer $(20 \mathrm{mM}$ Tris $\mathrm{pH}=8.0$, $400 \mathrm{mM} \mathrm{NaCl}, 10 \%$ glycerol, $2 \mathrm{mM}$ CHAPS, $5 \mathrm{mM}$ imidazole) and lysed by sonication. The crude extract was centrifuged at $45^{\prime} 000 \mathrm{~g}$ for 1 hour at $4^{\circ} \mathrm{C}$. The lysate was loaded on a Ni affinity step on HisTrap FF crude column (GE Healthcare, Inc.) and the protein was eluted at a concentration of $150 \mathrm{mM}$ imidazole. The LBD protein was then polished by size-exclusion chromatography in a SEC buffer ( $20 \mathrm{mM}$ Tris $\mathrm{pH}=8.0,250 \mathrm{mM} \mathrm{NaCl}, 2 \mathrm{mM}$ TCEP) by using a Superdex S75 16/60 column (GE Healthcare).

\section{Polyacrylamide native gel electrophoresis}

The individual proteins were run on an $8 \%$ polyacrylamide gel (PAGE) at $2 \mathrm{~W}$ constant power after pre-running the gel for $40 \mathrm{~min}$ at $4^{\circ} \mathrm{C}$. The native gel system was based on a Tris/CAPS $(\mathrm{pH}=9.4)$ buffer system that contained $60 \mathrm{mM}$ Tris base and $40 \mathrm{mM}$ CAPS (3-cyclohexilamino-1-propane-sulfonic acid). Approximately 3-5 $\mu \mathrm{g}$ protein was loaded per lane along 
with its DNA counterpart at defined molar ratios. The polyacrylamide gels were stained using Instant Blue Protein Stain (Expedeon Protein Solutions) for $15 \mathrm{~min}$ and rinsed in water.

\section{Analytical ultracentrifugation}

Sedimentation velocity experiments were conducted using Beckman Coulter ProteomeLab XL-I analytical ultracentrifuge using the 8-hole Beckman An-50Ti rotor at $4^{\circ} \mathrm{C}$ for samples in a buffer composed of $20 \mathrm{mM}$ Tris $\mathrm{pH}=8.0,250 \mathrm{mM} \mathrm{NaCl}, 20 \mu \mathrm{M}$ TCEP [72]. The molar protein concentration of the experiments corresponds to $1 \mu \mathrm{M}$. Sedimentation at $50000 \mathrm{rpm}$ was monitored by absorbance at $220 \mathrm{~nm}$ with scans made at $5 \mathrm{~min}$ intervals. The solution density and viscosity for resuspension buffer were calculated using SEDNTERP software. Data were analyzed using a c(s) model in SEDFIT [73].

\section{Size-exclusion chromatography hyphenated to non-denaturing mass spectrometry (SEC-non denaturing MS)}

For SEC-non-denaturing MS analysis, an ACQUITY UPLC H-class system (Waters, Manchester, UK) comprising a quaternary solvent manager, a sample manager cooled at $10^{\circ} \mathrm{C}$, a column oven maintained at room temperature and a TUV detector operating at $280 \mathrm{~nm}$ and 214 $\mathrm{nm}$ was coupled to the Synapt G2 HDMS mass spectrometer (Waters, Manchester, UK). $50 \mu \mathrm{g}$ of each samples were loaded on the ACQUITY UPLC Protein BEH SEC column $(4.6 \times 150$ $\mathrm{mm}, 1.7 \mu \mathrm{m}$ particle size, $200 \AA$ A pore size from Waters, Manchester, UK) using an isocratic elution of $150 \mathrm{mM}$ ammonium acetate $\left(\mathrm{NH}_{4} \mathrm{OAc}\right)$ at $\mathrm{pH} 7.4$ and at a flow rate of $0.25 \mathrm{~mL} / \mathrm{min}$ over $4.0 \mathrm{~min}$. Then the flow rate was decreased to $0.10 \mathrm{~mL} / \mathrm{min}$ over $5.9 \mathrm{~min}$ and finally increased to $0.25 \mathrm{~mL} / \mathrm{min}$ over $1.9 \mathrm{~min}$. The Synapt G2 HDMS was operated in positive mode with a capillary voltage of $3.0 \mathrm{kV}$ while sample cone and pressure in the interface region were set to $40 \mathrm{~V}$ and $6 \mathrm{mbar}$, respectively Acquisitions were performed in 1,000-10,000 m/z range with a $1.5 \mathrm{~s}$ scan time. The mass spectrometer was calibrated using singly charged ions produced by a $2 \mathrm{~g} / \mathrm{L}$ solution of cesium iodide (Acros organics, Thermo Fisher Scientific, Waltham, MA USA) in 2-propanol/water (50/50 v/v). Native MS data interpretations were performed using Mass Lynx V4.1 (Waters, Manchester, UK). For PPAR $\alpha / R X R \alpha$ experiments, the two nuclear receptors were expressed in E. coli and purified separately. Mixtures of 1:1 molar ratio of RXR $\alpha$ (wt or mutants) and PPAR $\alpha$ LBD were performed in the purification buffer, incubated and injected on the SEC column coupled to the mass spectrometry instrument as described above. Deconvolution was performed using UniDEc [74]. Relative abundances of the species were calculated from native MS intensities of the deconvoluted data.

\section{Off-line native electrospray-mass spectrometry (ESI-MS)}

Samples were first buffer exchanged in $150 \mathrm{mM}$ ammonium acetate $\left(\mathrm{NH}_{4} \mathrm{OAc}\right)$ at $\mathrm{pH} 7.4$ using $0.5 \mathrm{~mL}$ Zeba ${ }^{\mathrm{TM}}$ Spin desalting Columns (Thermo Fisher Scientific, Waltham, MA USA). Then, concentrations were determined by UV-Vis using a Nanodrop 2000 Spectrophotometer (Thermo Fisher Scientific, Waltham, MA USA). Finally, analyses were performed on an electrospray time-of-flight mass spectrometer (LCT, Waters, Manchester, UK). Samples were diluted to a monomeric protein concentration of $10 \mu \mathrm{M}$ and directly infused into the mass spectrometer via an automated chip-based nanoESI source (Triversa Nanomate, Advion, Ithaca, NY). Instrumental parameters were optimized for the detection of noncovalent complexes by raising the interface pressure to $6 \mathrm{mbar}$ and the cone voltage to $60 \mathrm{~V}$. Acquisitions were performed in 1,000-10,000 $\mathrm{m} / \mathrm{z}$ range with a $4 \mathrm{~s}$ scan time in positive mode The mass spectrometer was also calibrated using singly charged ions produced by a $2 \mathrm{~g} / \mathrm{L}$ solution of cesium iodide (Acros organics, Thermo Fisher Scientific, Waltham, MA USA) in 2-propanol/water (50/50 v/ 
v). Native MS data interpretations were performed using Mass Lynx V4.1 (Waters, Manchester, UK).

\section{Molecular modeling of sponge NR1}

Homology modeling of the NR1 sequence from Amphimedon queenslandica (ACA04755.1) using multiple templates was performed using Modeler (Webb and Sali, 2016) in order to evaluate the possible three-dimensional fold of this sequence, in particular whether a $\pi$-turn could be formed. The multiple alignment was constructed using 15 PDB structures of RXR (1MV9, 1H9U), USP (1Z5X, 1G2N, 1HG4), HNF4 (1LV2, 1PZL), LRH (1PK5, 1YUC), RAR (1DKF, 1FCY, 1XAP), TR (1NAV), ERR (1S9P) and LXR (1UPV). Sequence identity between NR1 sequence and these templates ranged from $27 \%$ (ERR) to $42 \%$ (RXR). A total of one hundred models were generated and evaluated according to their DOPE scores. The model with the best score, as well as all other models obtained contain a $\pi$-turn in helix $\mathrm{H} 7$, with very similar orientations of $\mathrm{R}$ and $\mathrm{E}$ residues when compared to RXR (obtained in the structure PDB code 1DKF). Since sequence identity is very elevated between NR1 and RXR and HNF4 sequences, another homology model was built using the same multiple alignment with only sequences of receptors without a $\pi$-turn and consisted of LRH (1PK5, 1YUC), RAR (1DKF, 1FCY, 1XAP), TR (1NAV), ERR (1S9P) and LXR (1UPV). Similarly, a total of one hundred models were calculated and their quality evaluated according to their DOPE scores. In this case, the best model does not contain a $\pi$-turn in H7, however when comparing the scores of both best models from the two modeling strategies, the model with $\pi$-turn has the best score (-27875.7) with respect to the one without a $\pi$-turn (-27771).

Model assessment was also calculated using ProQ2 (Ray et al., 2012), an algorithm predicting local and global quality of protein models, based on properties from sequence (predicted secondary structure for example) and structure (atom-atom contacts, residue-residue contacts, secondary structure). This algorithm provides a score for each residue and was used to assess the quality of the homology modeling specifically for helix H7. We calculated the average score of helix $\mathrm{H} 7$ (ranging from 0 to 1 , the latter being the best score) and obtained 0.71 and 0.65 with standard deviations of 0.06 and 0.04 for the models with and without $\pi$-turn respectively, supporting an enhancement in quality with the presence of the $\pi$-turn in the nuclear receptor structure.

Homology modeling using multiple templates was performed using Modeler (Webb and Sali, 2016), sequences with an e-value of 0 (best alignment) were extracted from non-redundant PDB sequences. The multiple alignment was constructed using 15 PDB structures of RXR (1MV9, 1H9U), USP (1Z5X, 1G2N, 1HG4), HNF4 (1LV2, 1PZL), LRH (1PK5, 1YUC), RAR (1DKF, 1FCY, 1XAP), TR (1NAV), ERR (1S9P) and LXR (1UPV). Sequence identity between NR1 sequence and these templates ranged from $27 \%$ (ERR) to $42 \%$ (RXR). A total of one hundred models were generated and evaluated according to their DOPE scores. Another homology modeling run was performed using structures without a $\pi$-turn in helix $\mathrm{H} 7$ and consisted of LRH (1PK5, 1YUC), RAR (1DKF, 1FCY, 1XAP), TR (1NAV), ERR (1S9P) and LXR (1UPV). Similarly, a total of one hundred models were calculated and their quality evaluated according to their DOPE scores.

\section{Molecular Dynamics simulations}

Simulations were performed with the LBD of RXR using the crystal structure 1MVC. Substitution of E352 residue by an alanine in the wild-type structure was performed using the mutagenesis wizard in the PyMol program (The PyMOL Molecular Graphics System VS, LLC.). Generation of the $\triangle \mathrm{E} 352$ was done using Modeler, providing the structure of RXR as template 
for the $\Delta \mathrm{E} 352$ sequence. A model without a $\pi$-turn was thus generated, and we insured that all other side chains aside from this region remained similar to the wild-type structure. Hydrogen atom placement was performed using the HBUILD facility [75] in the CHARMM program [76]. All three structures were solvated in cubic boxes of approximately 121 per side, with a salt concentration $\mathrm{Na}+/ \mathrm{Cl}$ - corresponding to the physiological concentration of $150 \mathrm{mM}$. Before solvating the system, two minimizations of 100 steps of Steepest Descent method and 1000 steps of Adapted Basis Newton-Raphson method were performed in order to eliminate steric clashes.

Molecular dynamics simulations were performed using the CHARMM36 force field [77] within the NAMD program [78], following two steps. First, minimization and heating of water molecules around the fixed protein was realized with 1000 steps of Conjugate Gradient (CG) energy minimization, heating up to $600 \mathrm{~K}$ over $23 \mathrm{ps}, 250$ steps of CG energy minimization, and heating to $300 \mathrm{~K}$ over $25 \mathrm{ps}$. Second, positional restraints on the protein were removed and all the system was energy minimized with 2000 steps of CG and heating to $300 \mathrm{~K}$ over $15 \mathrm{ps}$, followed by $85 \mathrm{ps}$ of equilibration. The production run was then performed for the duration of 50ns. Periodic boundary conditions were used and the particle mesh Ewald algorithm [79] was applied to take into account long-range electrostatic interactions. All bonds between heavy atoms and hydrogens were constrained using the SHAKE algorithm [80] and an integration time step of 2 fs was used for all simulations. This protocol has been carefully benchmarked across different nuclear receptor proteins, such as RAR, ER, GR [81-85]. The first 10ns of the production run were excluded from all analysis to ensure proper equilibration. Time evolution of $\mathrm{C}_{\alpha}$-RMSD of the three systems wt RXR $\alpha$ LBD, $\triangle \mathrm{E} 352 \mathrm{RXR} \alpha \mathrm{LBD}$, and E352A RXR $\alpha$ LBD across the three simulations are represented in S6D Fig to illustrate the stability of the structures over the course of the analyzed timeframe.

Binding free energies were estimated on the average structures, calculated over the time frame between 10 to 50ns. We used the University of Houston Brownian Dynamics (UHBD) [86], to solve the linearized Poisson-Boltzmann equation and compute the electrostatic binding free energy of binding of the two molecules. A dielectric constant of 80 was used for the solvent, 1 for the protein and a $\mathrm{pH}$ of 7 . Van der Waals radii and charges of atoms are obtained from the force field CHARMM36. A nonbonded cutoff of $12.5 \AA$ were used with a shift truncation function for electrostatics. Although MM/PBSA does not take into account conformational entropy, our protocol [87] has been validated on a variety of systems to assess protein/ protein complexes in terms of relative free energies and proven to be in agreement with experimental data $[84,85]$.

\section{Supporting information}

S1 Fig. Reference sequence alignment snippet used in this article. Alignment generated with Clustal Omega software and manually corrected.

S2 Fig. Helix $\mathbf{H 7}$ of the ligand-binding domain of COUP-TFII displays a double conformation. (A) Ribbon diagram showing the double conformation of helix H7. Conformation A is colored in green, and conformation $B$ in blue in all images. All images are presented in crosseye stereo. (B) $2 \mathrm{mFo}-\mathrm{DFc}$ electron density map contoured at 0.5 sigma $\left(0.16 \mathrm{e}-/ \AA^{3}\right)$ around helix H7. The density for conformation $B$ is shown in blue, and the supplemental density for conformation $\mathrm{A}$ is shown in green. (C) $2 \mathrm{mFo}-\mathrm{DFc}$ electron density map contoured at 0.5 sigma $\left(0.16 \mathrm{e}-/ \AA^{3}\right)$ around conformation B only of helix H7. (D) $2 \mathrm{mFo}-\mathrm{DFc}$ electron density map contoured at 0.5 sigma $\left(0.16 \mathrm{e}-/ \AA^{3}\right)$ around conformation A only of helix H7.

(TIF) 
S3 Fig. Maximum-likelihood phylogenetic tree of nuclear receptors. Classical and newly defined NR families are indicated with grey boxes. Species sequences are colored according to the five main metazoan groups they belong to. Branch support values are assessed by approximate likelihood ratio test (aLRT) and are show only for nodes that are considered fully robust (above 0.97). Occurrence of the RxxxE motif is also indicated. Our improved sampling also confirms that there are two cnidarian-specific NR groups. The first one is NR8, that branches at the basis of the crow group containing the bilaterian-specific NR1/NR4 group. The NR1/4/8 group is sister to the eumetazoan NR7 group, which has undergone lineage specific losses in vertebrates and arthropods, and is therefore represented here by sequences from the leech Helobdella robusta and Branchiostoma lanceolatum. Although the internal branching between NR1, N4, NR7 and NR8 are not supported by strong branch support, they are consistent with the last global phylogenetic analysis of the NR family [9]. In this study, the NR7 group was informally called "INRa" and the NR7 group was called "INRb". Also, there is an additional cnidarian-specific group branching at the basis of the bilaterian NR5 and NR6 groups, which was unformally called "cSF-1". Here it is named NR9, since it is at the basis of NR5 and NR6, and two additional sequences from the anthozoan cnidarians Pocillopora damicornis and Acropora millepora are provided. Concerning the NR3 family, cnidarian sequences were identified [88] and here, the Hydra sequence was used as a representative of this group. Interestingly, among basal groups, the loss of the RxxxE motif is always associated with other deviations from the ancestral state. In the NR2A group, the RxxxE motif has been lost in a group comprising the Ctenophoran sequences, that completely lost their DBD ((Reitzel et al., 2011); in blue, as well as in all of the sponges sequences, in which the DBD diverges through a 2 to 5 residue insert. Inside the sponge SpNR1 group, it was lost once in the paralog P3 group which exhibits the longest branch length, further indicating of a high substitution rate. In the NR2E group, the RxxxE motif is fully lost in the longest branch leading to Drosophila FAX-1, whereas among the other branches, there are already indications of higher structural variation. There is a KxxxE motif in Drosophila DSF and Nematostella 183874, a QxxxE motif in humain TLX, and an RxxxxxxE motif in Drosophila TLL. On the other hand, the RxxxE motif is conserved in the PNR group, for bilaterian and cnidarians, and also in the two NR2E1 sequences from cnidarians with the shortest branches (S2 Fig). In the NR2F group, only one cnidarian sequence, the one of Hydractinia echinata, is fully lost the RxxxE motif, while it is present as the KxxxE variant in two more cnidarian sequences. The 21656 sequences from the placozoan Trichoplax adhaerens, which branches separately from the eumetazoan NR2C/D/H, NR2E and NR2F group, illustrate the fact that early diverging organisms may also deviate from the ancestral pattern. Lastly, regarding the crown group containing NR5/6/9, NR3 and NR1/4/7/8, the Trichoplax NR3 sequence was the only one to keep the RxxxE motif.

S4 Fig. Oligomeric status of wild-type (wt) RXR $\alpha$ LBD and mutants, where the conserved Glu residue of the $\pi$-turn motif is mutated to Ala (E352A) or deleted $(\Delta \mathrm{E} 352)$. (A) Sizeexclusion chromatograms from analytical SEC chromatography on a S200 10/300 Superdex column for wt RXR $\alpha$ LBD (olive line), E352A RXR $\alpha$ LBD (cyan line) and $\Delta \mathrm{E} 352 \mathrm{RXR} \alpha \mathrm{LBD}$ (grey line). Peaks are named void for the peak roughly corresponding to the exclusion volume of the column, 'peak1 wt' and 'peak2 wt' for the two peaks of wt RXR $\alpha$ LBD and 'peak mut' for the peak of the mutant constructs. The peaks are further marked by symbols in colors that are then consistently used for the panel B; red for peak1 wt, blue for peak $2 \mathrm{wt}$, cyan for E352A $\mathrm{RXR} \alpha$ and grey for $\triangle \mathrm{E} 352 \mathrm{RXR} \alpha$. The polyacrylamide SDS gel depicted in the insert indicates the high purity of the samples. (B) Differential sedimentation coefficient distribution $\mathrm{c}(\mathrm{S})$ as a 
function of the sedimentation coefficient S (in Svedberg) obtained from AUC experiments at $1 \mu \mathrm{M}$, showing the $\mathrm{c}(\mathrm{S})$ distribution for peak $1 \mathrm{wt}$ (red curve) and peak $2 \mathrm{wt}$ ' (blue curve) of the wt RXR $\alpha$ LBD SEC peaks, as well as for peak mut of E352A RXR $\alpha$ LBD SEC peak (cyan curve) and $\Delta \mathrm{E} 352 \mathrm{RXR} \alpha \mathrm{LBD}$ SEC peak (grey curve).

(TIF)

S5 Fig. Oligomeric status of wild-type (wt) RXR $\alpha$ LBD and mutants, where the conserved Glu residue of the $\pi$-turn motif is mutated to Ala (E352A) or deleted ( $\triangle \mathrm{E} 352)$. (A-D) Native mass-spectrometric analysis of RXR LBD wt and mutants performed at a voltage $60 \mathrm{~V}$ of the SEC peaks. The inserts on the right show an enlarged view (by a factor of 6 ) of the $\mathrm{m} / \mathrm{z}$ region that encompasses the dimer $\mathrm{m} / \mathrm{z}$ peaks centered around the $16+$ species. Striking is the complete absence of tetramers for the RXR LBD mutant species. (E) Native polyacrylamide gel electrophoresis of the different SEC peaks of wt RXR $\alpha$ LBD (peak 1 and peak2) and of the mutant E352A RXR $\alpha$ LBD and $\triangle \mathrm{E} 352 \mathrm{RXR} \alpha \mathrm{LBD}$. T and $\mathrm{M}$ specifies the tetrameric and the monomeric species, respectively. (F) Cartoon representation of tetrameric RXR $\alpha$ as observed in the crystal structure of RXR $\alpha$ LBD (PDBcode 1G1U). The two homodimers of the tetramer (A1/B1 and A2/B2) are shown in yellow and cyan, respectively. The Arg and Glu residues of the $\pi$-turn are shown as sticks, colored in magenta for carbon, blue for nitrogen and red for oxygen. The triple Phe residues of $\mathrm{H} 11$ are depicted in as sticks colored in grey for carbon. Important helices of the tetramer interface are indicated. (TIF)

S6 Fig. Electrostatic interactions for the RXR wt and mutant homodimers. The electrostatics interactions were analyzed during the MD simulations of (A) wt, (B) E352A and (C) $\triangle E 352$ RXRa LBD homodimer complex. The percentage of interaction presence is represented with lines connecting the corresponding residues, in red (between 75 and $100 \%$ of simulation time), purple (between 50 and $75 \%$ of simulation time) and pink (between 25 and $50 \%$ of simulation time). (D) Time evolution of the Ca-RMSD of the wt RXR $\alpha$ LBD (black line), E352A $\mathrm{RXR} \alpha \mathrm{LBD}$ (blue line) and $\triangle \mathrm{E} 352 \mathrm{RXR} \alpha \mathrm{LBD}$ (red line), represented in black, red and blue respectively, over the course of the 50ns molecular dynamics simulations.

S7 Fig. Heterodimeric dimerization interface for EcR/USP-RXR of different species. (A-B) Detailed views of heterodimeric interactions between EcR and USP-RXR LBD structures of different insect species, the more recent species Heliothis virescens $(\mathrm{Hv})$, and the more basal species Tribolium castaneum (Tc) and Bemisia tabaci (Bt). The structure of HvEcR/USP-RXR is the one bound to 20-hydroxyecdysone (PDBcode 2R40), that of TcEcR/USP-RXR is bound to ponasterone $\mathrm{A}$ (PDBcode $2 \mathrm{NXX}$ ), that of $\mathrm{BtEcR} / \mathrm{USP}-\mathrm{RXR}$ is bound to ponasterone $\mathrm{A}$ (PDBcode 1Z5X). In (A), the view shows the interactions of USP-RXR H7 with the EcR partner loop H8-H9 which are rather conserved among species. In (B), the view shows the large differences in interaction distances between EcR H7 helix and the partner USP-RXR at the level of its loop H8-H9. Intermolecular interactions between EcR H7 and USP-RXR H8-H9 loop can only be formed for HvEcR/USP-RXR and not for TcEcR/USP-RXR and BtEcR/ USP-RXR, due to too large distances between these structural elements.

S8 Fig. Heterodimerization capacity of wild-type (wt) RXR $\alpha$ LBD and mutant E352A and $\triangle$ E352 RXR LBD with PPARalpha. Size-exclusion chromatography (SEC)-coupled native mass spectrometric (MS) analysis for complex mixture of PPAR $\alpha$ LBD with either (A) wt RXR $\alpha$ LBD, (B) E352A RXR $\alpha$ LBD or (C) $\triangle \mathrm{E} 352 \mathrm{RXR} \alpha \mathrm{LBD}$. The isolated RXR $\alpha$ LBD (wt or mutants) is depicted in yellow, isolated PPAR $\alpha$ LBD in blue and heterodimeric PPAR $\alpha / R X R \alpha$ 
LBDs in green. The raw mass spectra are depicted together with the size-exclusion chromatograms of wt RXR $\alpha$ LBD and of mutants RXR $\alpha$ LBD. Integration of the mass spectra is performed over the whole peak of the chromatogram.

(TIF)

S1 Table. Data collection and refinement statistics. For the original structure, statistics for the highest resolution shell are given in parentheses. For the original structure reported statistics are shown in red, and recalculated statistics in blue.

(XLSX)

S2 Table. Molecular masses measured in the size-exclusion chromatography (SEC)-coupled native mass spectrometric analysis. Molecular masses are given for the two SEC-MS peaks seen for wt RXR $\alpha$ LBD and for the single peak observed for the mutants E352A RXR $\alpha$ $\mathrm{LBD}$ and $\triangle \mathrm{E} 352 \mathrm{RXR} \alpha \mathrm{LBD}$. The region of the SEC-MS peaks that were integrated for mass determination are shown in the insert of Fig $6 \mathrm{~B}$ by a line over the peaks.

(XLSX)

S3 Table. Molecular masses measured in the native mass spectrometric analysis. Molecular masses are given for the different SEC peaks collected during the analytical SEC analysis shown in Fig 6A.

(XLSX)

S4 Table. Total binding free energy from Molecular Dynamics (MD) simulations. Shown is the total binding free energy between each RXR subunit of the homodimer made of wt RXR $\alpha$ $\mathrm{LBD}, \mathrm{E} 352 \mathrm{~A} \mathrm{RXR} \alpha \mathrm{LBD}$ and $\triangle \mathrm{E} 352 \mathrm{RXR} \alpha \mathrm{LBD}$, respectively, calculated from $50 \mathrm{~ns} \mathrm{MD}$ simulations.

(XLSX)

\section{Acknowledgments}

The authors would like to thank Bariza Blanquier, manager of Genetic Analysis facility for her expert assistance (AniRA platform, SFR BioSciences Gerland-Lyon Sud UMS3444/US8). We would like to acknowledge the High-Performance Computing Center of the Université de Strasbourg for providing access to computing resources. We thank I. Hazemann for excellent technical assistance, Anthony Ehkirch for SEC-MS analysis, C. Birck for AUC experiments, K. Essabri for mutagenesis of HsRXR LBD, B. Klaholz and A. Dejaegere for discussion and François Bonneton for review and comments. We thank Bruno Frédérich (Université de Liège) for help in using phytools.

\section{Author Contributions}

Conceptualization: Brice Beinsteiner, Gabriel V. Markov, Vincent Laudet, Dino Moras, Isabelle M. L. Billas.

Data curation: Brice Beinsteiner, Gabriel V. Markov, Yassmine Chebaro, Alastair G. McEwen, Isabelle M. L. Billas.

Formal analysis: Brice Beinsteiner, Gabriel V. Markov, Alastair G. McEwen, Sarah Cianférani, Vincent Laudet, Dino Moras, Isabelle M. L. Billas.

Funding acquisition: Sarah Cianférani, Vincent Laudet, Dino Moras, Isabelle M. L. Billas.

Investigation: Brice Beinsteiner, Gabriel V. Markov, Stéphane Erb, Yassmine Chebaro, Alastair G. McEwen, Isabelle M. L. Billas. 
Methodology: Brice Beinsteiner, Gabriel V. Markov, Yassmine Chebaro, Sarah Cianférani, Isabelle M. L. Billas.

Project administration: Vincent Laudet, Dino Moras, Isabelle M. L. Billas.

Resources: Brice Beinsteiner, Gabriel V. Markov, Sarah Cianférani.

Software: Brice Beinsteiner, Gabriel V. Markov, Yassmine Chebaro.

Supervision: Sarah Cianférani, Vincent Laudet, Dino Moras, Isabelle M. L. Billas.

Validation: Brice Beinsteiner, Gabriel V. Markov, Yassmine Chebaro, Sarah Cianférani, Vincent Laudet, Dino Moras, Isabelle M. L. Billas.

Visualization: Brice Beinsteiner, Gabriel V. Markov, Alastair G. McEwen, Sarah Cianférani, Vincent Laudet, Dino Moras, Isabelle M. L. Billas.

Writing - original draft: Brice Beinsteiner, Gabriel V. Markov, Vincent Laudet, Dino Moras, Isabelle M. L. Billas.

Writing - review \& editing: Brice Beinsteiner, Gabriel V. Markov, Sarah Cianférani, Vincent Laudet, Dino Moras, Isabelle M. L. Billas.

\section{References}

1. Billas I, Moras D. Allosteric Controls of Nuclear Receptor Function in the Regulation of Transcription. J Mol Biol. 2013; 425: 2317-2329. https://doi.org/10.1016/j.jmb.2013.03.017 PMID: 23499886

2. Gronemeyer H, Gustafsson J-A, Laudet V. Principles for modulation of the nuclear receptor superfamily. Nat Rev Drug Discov. 2004; 3: 950-964. https://doi.org/10.1038/nrd1551 PMID: 15520817

3. Lazar MA. Maturing of the nuclear receptor family. J Clin Invest. 2017; 127: 1123-1125. https://doi.org/ 10.1172/JCI92949 PMID: 28368290

4. Amero SA, Kretsinger RH, Moncrief ND, Yamamoto KR, Pearson WR. The origin of nuclear receptor proteins: a single precursor distinct from other transcription factors. Mol Endocrinol Baltim Md. 1992; 6: 3-7. https://doi.org/10.1210/mend.6.1.1738368 PMID: 1738368

5. Escriva H, Safi R, Hänni C, Langlois M-C, Saumitou-Laprade P, Stehelin D, et al. Ligand binding was acquired during evolution of nuclear receptors. Proc Natl Acad Sci. 1997; 94: 6803-6808. https://doi. org/10.1073/pnas.94.13.6803 PMID: 9192646

6. Laudet V, Hänni C, Coll J, Catzeflis F, Stéhelin D. Evolution of the nuclear receptor gene superfamily. EMBO J. 1992; 11: 1003-1013. PMID: 1312460

7. López-Escardó D, Grau-Bové X, Guillaumet-Adkins A, Gut M, Sieracki ME, Ruiz-Trillo I. Reconstruction of protein domain evolution using single-cell amplified genomes of uncultured choanoflagellates sheds light on the origin of animals. Philos Trans R Soc Lond B Biol Sci. 2019; 374: 20190088. https://doi.org/ 10.1098/rstb.2019.0088 PMID: 31587642

8. Bertrand S, Brunet FG, Escriva H, Parmentier G, Laudet V, Robinson-Rechavi M. Evolutionary Genomics of Nuclear Receptors: From Twenty-Five Ancestral Genes to Derived Endocrine Systems. Mol Biol Evol. 2004; 21: 1923-1937. https://doi.org/10.1093/molbev/msh200 PMID: 15229292

9. Bridgham JT, Eick GN, Larroux C, Deshpande K, Harms MJ, Gauthier MEA, et al. Protein Evolution by Molecular Tinkering: Diversification of the Nuclear Receptor Superfamily from a Ligand-Dependent Ancestor. PLoS Biol. 2010; 8: e1000497. https://doi.org/10.1371/journal.pbio.1000497 PMID: 20957188

10. Robinson-Rechavi M, Maina CV, Gissendanner CR, Laudet V, Sluder A. Explosive Lineage-Specific Expansion of the Orphan Nuclear Receptor HNF4 in Nematodes. J Mol Evol. 2005; 60: 577-586. https://doi.org/10.1007/s00239-004-0175-8 PMID: 15983867

11. Simion P, Philippe H, Baurain D, Jager M, Richter DJ, Di Franco A, et al. A Large and Consistent Phylogenomic Dataset Supports Sponges as the Sister Group to All Other Animals. Curr Biol CB. 2017; 27: 958-967. https://doi.org/10.1016/j.cub.2017.02.031 PMID: 28318975

12. Eick GN, Thornton JW. Evolution of steroid receptors from an estrogen-sensitive ancestral receptor. Mol Cell Endocrinol. 2011; 334: 31-38. https://doi.org/10.1016/j.mce.2010.09.003 PMID: 20837101

13. Markov GV, Laudet V. Origin and evolution of the ligand-binding ability of nuclear receptors. Mol Cell Endocrinol. 2011; 334: 21-30. https://doi.org/10.1016/j.mce.2010.10.017 PMID: 21055443 
14. Germain P, Bourguet W. Dimerization of nuclear receptors. Methods Cell Biol. 2013; 117: 21-41. https://doi.org/10.1016/B978-0-12-408143-7.00002-5 PMID: 24143970

15. Brélivet $\mathrm{Y}$, Rochel N, Moras $\mathrm{D}$. Structural analysis of nuclear receptors: From isolated domains to integral proteins. Mol Cell Endocrinol. 2012; 348: 466-473. https://doi.org/10.1016/j.mce.2011.08.015 PMID: 21888944

16. Khorasanizadeh S, Rastinejad F. Nuclear-receptor interactions on DNA-response elements. Trends Biochem Sci. 2001; 26: 384-390. https://doi.org/10.1016/s0968-0004(01)01800-x PMID: 11406412

17. Brelivet $\mathrm{Y}, \mathrm{Kammerer} \mathrm{S}$, Rochel N, Poch $\mathrm{O}$, Moras D. Signature of the oligomeric behaviour of nuclear receptors at the sequence and structural level. EMBO Rep. 2004; 5: 423-429. https://doi.org/10.1038/ sj.embor.7400119 PMID: 15105832

18. Bourguet $\mathrm{W}$, Ruff $\mathrm{M}$, Chambon $\mathrm{P}$, Gronemeyer $\mathrm{H}$, Moras $\mathrm{D}$. Crystal structure of the ligand-binding domain of the human nuclear receptor RXR- $\alpha$. Nature. 1995; 375: 377-382. https://doi.org/10.1038/ 375377a0 PMID: 7760929

19. Eberhardt J, McEwen AG, Bourguet W, Moras D, Dejaegere A. A revisited version of the apo structure of the ligand-binding domain of the human nuclear receptor retinoic $X$ receptor $\alpha$. Acta Crystallogr Sect F Struct Biol Commun. 2019; 75: 98-104. https://doi.org/10.1107/S2053230X18018022 PMID: 30713160

20. Billas IM, Moulinier L, Rochel N, Moras D. Crystal structure of the ligand-binding domain of the ultraspiracle protein USP, the ortholog of retinoid X receptors in insects. J Biol Chem. 2001; 276: 74657474. https://doi.org/10.1074/jbc.M008926200 PMID: 11053444

21. Wisely GB, Miller AB, Davis RG, Thornquest AD, Johnson R, Spitzer T, et al. Hepatocyte nuclear factor 4 is a transcription factor that constitutively binds fatty acids. Struct Lond Engl 1993. 2002; 10: 12251234. https://doi.org/10.1016/s0969-2126(02)00829-8 PMID: 12220494

22. Cartailler J-P, Luecke $H$. Structural and Functional Characterization of $\pi$ Bulges and Other Short Intrahelical Deformations. Structure. 2004; 12: 133-144. https://doi.org/10.1016/j.str.2003.12.001 PMID: 14725773

23. Cooley RB, Arp DJ, Karplus PA. Evolutionary Origin of a Secondary Structure: $\pi-H e l i c e s$ as Cryptic but Widespread Insertional Variations of $\alpha$-Helices That Enhance Protein Functionality. J Mol Biol. 2010; 404: 232-246. https://doi.org/10.1016/j.jmb.2010.09.034 PMID: 20888342

24. Kumar P, Bansal M. Dissecting $\pi$-helices: sequence, structure and function. FEBS J. 2015; 282: 44154432. https://doi.org/10.1111/febs.13507 PMID: 26370783

25. Ludwiczak J, Winski A, da Silva Neto AM, Szczepaniak K, Alva V, Dunin-Horkawicz S. PiPred-a deeplearning method for prediction of $\pi$-helices in protein sequences. Sci Rep. 2019; 9: 6888. https://doi. org/10.1038/s41598-019-43189-4 PMID: 31053765

26. Riek RP, Graham RM. The elusive m-helix. J Struct Biol. 2011; 173: 153-160. https://doi.org/10.1016/j, jsb.2010.09.001 PMID: 20828621

27. Watkins RE, Wisely GB, Moore LB, Collins JL, Lambert MH, Williams SP, et al. The human nuclear xenobiotic receptor PXR: structural determinants of directed promiscuity. Science. 2001; 292: 23292333. https://doi.org/10.1126/science.1060762 PMID: 11408620

28. Blind RD, Sablin EP, Kuchenbecker KM, Chiu H-J, Deacon AM, Das D, et al. The signaling phospholipid PIP3 creates a new interaction surface on the nuclear receptor SF-1. Proc Natl Acad Sci U S A. 2014; 111: 15054-15059. https://doi.org/10.1073/pnas.1416740111 PMID: 25288771

29. Kruse SW, Suino-Powell K, Zhou XE, Kretschman JE, Reynolds R, Vonrhein C, et al. Identification of COUP-TFII Orphan Nuclear Receptor as a Retinoic Acid-Activated Receptor. PLoS Biol. 2008; 6: e227. https://doi.org/10.1371/journal.pbio.0060227 PMID: 18798693

30. Tan MHE, Zhou XE, Soon F-F, Li X, Li J, Yong E-L, et al. The Crystal Structure of the Orphan Nuclear Receptor NR2E3/PNR Ligand Binding Domain Reveals a Dimeric Auto-Repressed Conformation. PLoS ONE. 2013; 8. https://doi.org/10.1371/journal.pone.0074359 PMID: 24069298

31. Gronemeyer H, Laudet V. Transcription factors 3: nuclear receptors. Protein Profile. 1995; 2: 11731308. PMID: 8681033

32. Adelmant $G$, Bègue A, Stéhelin D, Laudet V. A functional Rev-erb alpha responsive element located in the human Rev-erb alpha promoter mediates a repressing activity. Proc Natl Acad Sci U S A. 1996; 93 : 3553-3558. https://doi.org/10.1073/pnas.93.8.3553 PMID: 8622974

33. Alexander SPH, Cidlowski JA, Kelly E, Mathie A, Peters JA, Veale EL, et al. THE CONCISE GUIDE TO PHARMACOLOGY 2019/20: Nuclear hormone receptors. Br J Pharmacol. 2019; 176: S229-S246. https://doi.org/10.1111/bph.14750 PMID: 31710718

34. Lanz RB, Jericevic Z, Zuercher WJ, Watkins C, Steffen DL, Margolis R, et al. Nuclear Receptor Signaling Atlas (www.nursa.org): hyperlinking the nuclear receptor signaling community. Nucleic Acids Res. 2006; 34: D221-226. https://doi.org/10.1093/nar/gkj029 PMID: 16381851 
35. Laudet $\mathrm{V}$. Evolution of the nuclear receptor superfamily: early diversification from an ancestral orphan receptor. J Mol Endocrinol. 1997; 19: 207-226. https://doi.org/10.1677/jme.0.0190207 PMID: 9460643

36. Ochsner SA, Abraham D, Martin K, Ding W, McOwiti A, Kankanamge W, et al. The Signaling Pathways Project, an integrated 'omics knowledgebase for mammalian cellular signaling pathways. Sci Data. 2019; 6: 252. https://doi.org/10.1038/s41597-019-0193-4 PMID: 31672983

37. Kojetin DJ, Matta-Camacho E, Hughes TS, Srinivasan S, Nwachukwu JC, Cavett V, et al. Structural mechanism for signal transduction in RXR nuclear receptor heterodimers. Nat Commun. 2015; 6: 8013. https://doi.org/10.1038/ncomms9013 PMID: 26289479

38. Eeckhoute J, Oxombre B, Formstecher P, Lefebvre P, Laine B. Critical role of charged residues in helix 7 of the ligand binding domain in Hepatocyte Nuclear Factor 4alpha dimerisation and transcriptional activity. Nucleic Acids Res. 2003; 31: 6640-6650. https://doi.org/10.1093/nar/gkg850 PMID: 14602925

39. Cooper DN, Ball EV, Krawczak M. The human gene mutation database. Nucleic Acids Res. 1998; 26 : 285-287. https://doi.org/10.1093/nar/26.1.285 PMID: 9399854

40. Ehkirch A, Hernandez-Alba O, Colas O, Beck A, Guillarme D, Cianférani S. Hyphenation of size exclusion chromatography to native ion mobility mass spectrometry for the analytical characterization of therapeutic antibodies and related products. J Chromatogr B Analyt Technol Biomed Life Sci. 2018; 1086 : 176-183. https://doi.org/10.1016/j.jchromb.2018.04.010 PMID: 29684909

41. Kersten S, Reczek PR, Noy N. The tetramerization region of the retinoid X receptor is important for transcriptional activation by the receptor. J Biol Chem. 1997; 272: 29759-29768. https://doi.org/10.1074/ jbc.272.47.29759 PMID: 9368046

42. Kersten $S$, Kelleher $D$, Chambon $P$, Gronemeyer $H$, Noy $N$. Retinoid $X$ receptor alpha forms tetramers in solution. Proc Natl Acad Sci U S A. 1995; 92: 8645-8649. https://doi.org/10.1073/pnas.92.19.8645 PMID: 7567990

43. Borel F, de Groot A, Juillan-Binard C, de Rosny E, Laudet V, Pebay-Peyroula E, et al. Crystal structure of the ligand-binding domain of the retinoid $\mathrm{X}$ receptor from the ascidian Polyandrocarpa misakiensis. Proteins. 2009; 74: 538-542. https://doi.org/10.1002/prot.22294 PMID: 19004016

44. Gampe RT, Montana VG, Lambert MH, Wisely GB, Milburn MV, Xu HE. Structural basis for autorepression of retinoid X receptor by tetramer formation and the AF-2 helix. Genes Dev. 2000; 14: 2229-2241. https://doi.org/10.1101/gad.802300 PMID: 10970886

45. Wurtz JM, Bourguet W, Renaud JP, Vivat V, Chambon P, Moras D, et al. A canonical structure for the ligand-binding domain of nuclear receptors. Nat Struct Biol. 1996; 3: 87-94. https://doi.org/10.1038/ nsb0196-87 PMID: 8548460

46. Egea PF, Mitschler A, Moras D. Molecular Recognition of Agonist Ligands by RXRs. Mol Endocrinol. 2002; 16: 987-997. https://doi.org/10.1210/mend.16.5.0823 PMID: 11981034

47. Bourguet $\mathrm{W}$, Germain $\mathrm{P}$, Gronemeyer $\mathrm{H}$. Nuclear receptor ligand-binding domains: three-dimensional structures, molecular interactions and pharmacological implications. Trends Pharmacol Sci. 2000; 21: 381-388. https://doi.org/10.1016/s0165-6147(00)01548-0 PMID: 11050318

48. Bourguet W, Vivat V, Wurtz J-M, Chambon P, Gronemeyer H, Moras D. Crystal Structure of a Heterodimeric Complex of RAR and RXR Ligand-Binding Domains. Mol Cell. 2000; 5: 289-298. https://doi.org/ 10.1016/s1097-2765(00)80424-4 PMID: 10882070

49. Iwema $\mathrm{T}$, Billas IM, Beck $\mathrm{Y}$, Bonneton $\mathrm{F}$, Nierengarten $\mathrm{H}$, Chaumot $\mathrm{A}$, et al. Structural and functional characterization of a novel type of ligand-independent RXR-USP receptor. EMBO J. 2007; 26: 37703782. https://doi.org/10.1038/sj.emboj.7601810 PMID: 17673910

50. Maletta M, Orlov I, Roblin P, Beck Y, Moras D, Billas IML, et al. The palindromic DNA-bound USP/EcR nuclear receptor adopts an asymmetric organization with allosteric domain positioning. Nat Commun. 2014; 5. https://doi.org/10.1038/ncomms5139 PMID: 24942373

51. Ren B, Peat TS, Streltsov VA, Pollard M, Fernley R, Grusovin J, et al. Unprecedented conformational flexibility revealed in the ligand-binding domains of the Bovicola ovis ecdysone receptor $(\mathrm{EcR})$ and ultraspiracle (USP) subunits. Acta Crystallogr D Biol Crystallogr. 2014; 70: 1954-1964. https://doi.org/10. 1107/S1399004714009626 PMID: 25004972

52. Iwema T, Chaumot A, Studer RA, Robinson-Rechavi M, Billas IML, Moras D, et al. Structural and evolutionary innovation of the heterodimerization interface between USP and the ecdysone receptor ECR in insects. Mol Biol Evol. 2009; 26: 753-768. https://doi.org/10.1093/molbev/msn302 PMID: 19126866

53. Bonneton $F$, Chaumot $A$, Laudet $V$. Annotation of Tribolium nuclear receptors reveals an increase in evolutionary rate of a network controlling the ecdysone cascade. Insect Biochem Mol Biol. 2008; 38 : 416-429. https://doi.org/10.1016/j.ibmb.2007.10.006 PMID: 18342247

54. Chaumot A, Da Lage J-L, Maestro O, Martin D, Iwema T, Brunet F, et al. Molecular adaptation and resilience of the insect's nuclear receptor USP. BMC Evol Biol. 2012; 12: 199. https://doi.org/10.1186/14712148-12-199 PMID: 23039844 
55. Williams SP, Sigler PB. Atomic structure of progesterone complexed with its receptor. Nature. 1998; 393: 392-396. https://doi.org/10.1038/30775 PMID: 9620806

56. Krężel W, Rühl R, de Lera AR. Alternative retinoid X receptor (RXR) ligands. Mol Cell Endocrinol. 2019; 491: 110436. https://doi.org/10.1016/j.mce.2019.04.016 PMID: 31026478

57. Yuan X, Ta TC, Lin M, Evans JR, Dong Y, Bolotin E, et al. Identification of an endogenous ligand bound to a native orphan nuclear receptor. PloS One. 2009; 4: e5609. https://doi.org/10.1371/journal.pone. 0005609 PMID: 19440305

58. Grebner C, Lecina D, Gil V, Ulander J, Hansson P, Dellsen A, et al. Exploring Binding Mechanisms in Nuclear Hormone Receptors by Monte Carlo and X-ray-derived Motions. Biophys J. 2017; 112: 1147 1156. https://doi.org/10.1016/j.bpj.2017.02.004 PMID: 28355542

59. Billas IML, Iwema T, Garnier J-M, Mitschler A, Rochel N, Moras D. Structural adaptability in the ligandbinding pocket of the ecdysone hormone receptor. Nature. 2003; 426: 91-96. https://doi.org/10.1038/ nature02112 PMID: 14595375

60. Nettles KW, Bruning JB, Gil G, O'Neill EE, Nowak J, Hughs A, et al. Structural plasticity in the oestrogen receptor ligand-binding domain. EMBO Rep. 2007; 8: 563-568. https://doi.org/10.1038/sj.embor. 7400963 PMID: 17468738

61. Belorusova AY, Rochel N. Structural Studies of Vitamin D Nuclear Receptor Ligand-Binding Properties. Vitam Horm. 2016; 100: 83-116. https://doi.org/10.1016/bs.vh.2015.10.003 PMID: 26827949

62. Ciesielski F, Rochel N, Moras D. Adaptability of the Vitamin D nuclear receptor to the synthetic ligand Gemini: remodelling the LBP with one side chain rotation. J Steroid Biochem Mol Biol. 2007; 103: 235242. https://doi.org/10.1016/j.jsbmb.2006.12.003 PMID: 17218092

63. Delfosse V, Grimaldi M, Pons J-L, Boulahtouf A, le Maire A, Cavailles V, et al. Structural and mechanistic insights into bisphenols action provide guidelines for risk assessment and discovery of bisphenol $A$ substitutes. Proc Natl Acad Sci U S A. 2012; 109: 14930-14935. https://doi.org/10.1073/pnas. 1203574109 PMID: 22927406

64. Cuénot L. Théorie de la Préadaptation. Scientia. 1914; 8: 60.

65. Gould SJ, Vrba ES. Exaptation—a Missing Term in the Science of Form. Paleobiology. 1982; 8: 4-15. https://doi.org/10.1017/S0094837300004310

66. Gavelis GS, Keeling PJ, Leander BS. How exaptations facilitated photosensory evolution: Seeing the light by accident. BioEssays News Rev Mol Cell Dev Biol. 2017; 39. https://doi.org/10.1002/bies. 201600266 PMID: 28570771

67. Cornelis G, Vernochet C, Carradec Q, Souquere S, Mulot B, Catzeflis F, et al. Retroviral envelope gene captures and syncytin exaptation for placentation in marsupials. Proc Natl Acad Sci U S A. 2015; 112: E487-496. https://doi.org/10.1073/pnas.1417000112 PMID: 25605903

68. Maex M, Treer D, De Greve H, Proost P, Van Bocxlaer I, Bossuyt F. Exaptation as a Mechanism for Functional Reinforcement of an Animal Pheromone System. Curr Biol CB. 2018; 28: 2955-2960.e5. https://doi.org/10.1016/j.cub.2018.06.074 PMID: 30197090

69. Force A, Lynch M, Pickett FB, Amores A, Yan YL, Postlethwait J. Preservation of duplicate genes by complementary, degenerative mutations. Genetics. 1999; 151: 1531-1545. PMID: 10101175

70. Plach MG, Reisinger B, Sterner R, MerkI R. Long-Term Persistence of Bi-functionality Contributes to the Robustness of Microbial Life through Exaptation. PLoS Genet. 2016; 12: e1005836. https://doi.org/ 10.1371/journal.pgen.1005836 PMID: 26824644

71. Yanai I, Yu Y, Zhu X, Cantor CR, Weng Z. An avidin-like domain that does not bind biotin is adopted for oligomerization by the extracellular mosaic protein fibropellin. Protein Sci Publ Protein Soc. 2005; 14: 417-423. https://doi.org/10.1110/ps.04898705 PMID: 15659374

72. Lebowitz J, Lewis MS, Schuck P. Modern analytical ultracentrifugation in protein science: a tutorial review. Protein Sci Publ Protein Soc. 2002; 11: 2067-2079. https://doi.org/10.1110/ps.0207702 PMID: 12192063

73. Schuck $P$. Size-distribution analysis of macromolecules by sedimentation velocity ultracentrifugation and lamm equation modeling. Biophys J. 2000; 78: 1606-1619. https://doi.org/10.1016/S0006-3495 (00)76713-0 PMID: 10692345

74. Marty MT, Baldwin AJ, Marklund EG, Hochberg GKA, Benesch JLP, Robinson CV. Bayesian Deconvolution of Mass and Ion Mobility Spectra: From Binary Interactions to Polydisperse Ensembles. Anal Chem. 2015; 87: 4370-4376. https://doi.org/10.1021/acs.analchem.5b00140 PMID: 25799115

75. Brünger AT, Karplus M. Polar hydrogen positions in proteins: empirical energy placement and neutron diffraction comparison. Proteins. 1988; 4: 148-156. https://doi.org/10.1002/prot.340040208 PMID: 3227015 
76. Brooks BR, Brooks CL, Mackerell AD, Nilsson L, Petrella RJ, Roux B, et al. CHARMM: the biomolecular simulation program. J Comput Chem. 2009; 30: 1545-1614. https://doi.org/10.1002/jcc.21287 PMID: 19444816

77. Best RB, Zhu X, Shim J, Lopes PEM, Mittal J, Feig M, et al. Optimization of the additive CHARMM allatom protein force field targeting improved sampling of the backbone $\varphi, \psi$ and side-chain $\chi(1)$ and $\chi(2)$ dihedral angles. J Chem Theory Comput. 2012; 8: 3257-3273. https://doi.org/10.1021/ct300400x PMID: 23341755

78. Phillips JC, Braun R, Wang W, Gumbart J, Tajkhorshid E, Villa E, et al. Scalable molecular dynamics with NAMD. J Comput Chem. 2005; 26: 1781-1802. https://doi.org/10.1002/jcc.20289 PMID: 16222654

79. Darden T, York D, Pedersen L. Particle mesh Ewald: An N.log(N) method for Ewald sums in large systems. J Chem Phys. 1993; 98: 10089-10092. https://doi.org/10.1063/1.464397

80. Ryckaert J, Ciccotti G, Berendsen HJC. Numerical integration of the Cartesian equations of motion of a system with constraints: molecular dynamics of n-alkanes. J Comput Phys. 1977; 327-341.

81. Bianchetti L, Wassmer B, Defosset A, Smertina A, Tiberti ML, Stote RH, et al. Alternative dimerization interfaces in the glucocorticoid receptor- $\alpha$ ligand binding domain. Biochim Biophys Acta Gen Subj. 2018; 1862: 1810-1825. https://doi.org/10.1016/j.bbagen.2018.04.022 PMID: 29723544

82. Chebaro Y, Sirigu S, Amal I, Lutzing R, Stote RH, Rochette-Egly C, et al. Allosteric Regulation in the Ligand Binding Domain of Retinoic Acid Receptory. PLOS ONE. 2017; 12: e0171043. https://doi.org/ 10.1371/journal.pone.0171043 PMID: 28125680

83. Chebaro Y, Amal I, Rochel N, Rochette-Egly C, Stote RH, Dejaegere A. Phosphorylation of the Retinoic Acid Receptor Alpha Induces a Mechanical Allosteric Regulation and Changes in Internal Dynamics. PLOS Comput Biol. 2013; 9: e1003012. https://doi.org/10.1371/journal.pcbi.1003012 PMID: 23637584

84. Moroy G, Martin E, Dejaegere A, Stote $\mathrm{RH}$. Molecular basis for $\mathrm{Bcl}-2$ homology 3 domain recognition in the Bcl-2 protein family: identification of conserved hot spot interactions. J Biol Chem. 2009; 284: 17499-17511. https://doi.org/10.1074/jbc.M805542200 PMID: 19293158

85. Zanier K, Charbonnier S, Sidi AOMO, McEwen AG, Ferrario MG, Poussin-Courmontagne P, et al. Structural basis for hijacking of cellular LxxLL motifs by papillomavirus E6 oncoproteins. Science. 2013; 339: 694-698. https://doi.org/10.1126/science.1229934 PMID: 23393263

86. Madura JD, Briggs JM, Wade RC, Davis ME, Luty BA, llin A, et al. Electrostatics and diffusion of molecules in solution: simulations with the University of Houston Brownian Dynamics program. Comput Phys Commun. 1995; 91: 57-95. https://doi.org/10.1016/0010-4655(95)00043-F

87. Lafont $\mathrm{V}$, Schaefer $\mathrm{M}$, Stote $\mathrm{RH}$, Altschuh D, Dejaegere A. Protein-protein recognition and interaction hot spots in an antigen-antibody complex: free energy decomposition identifies "efficient amino acids." Proteins. 2007; 67: 418-434. https://doi.org/10.1002/prot.21259 PMID: 17256770

88. Khalturin K, Billas IML, Chebaro Y, Reitzel AM, Tarrant AM, Laudet V, et al. NR3E receptors in cnidarians: A new family of steroid receptor relatives extends the possible mechanisms for ligand binding. $J$ Steroid Biochem Mol Biol. 2018; 184: 11-19. https://doi.org/10.1016/j.jsbmb.2018.06.014 PMID: 29940311 